\title{
Spatially heterogeneous dynamics investigated via a time-dependent four-point density correlation function
}

\author{
N. Lačević \\ Department of Chemical Engineering, University of Michigan, Ann Arbor, Michigan 48109 and Department \\ of Physics and Astronomy, Johns Hopkins University, Baltimore, Maryland 21210 \\ F. W. Starr \\ Center for Theoretical and Computational Materials Science and Polymers Division, \\ National Institute of Standards and Technology, Gaithersburg, Maryland 20899
}

T. B. Schrøder

IMFUFA, Roskilde University, DK-4000, Denmark

\author{
S. C. Glotzer \\ Departments of Chemical Engineering and Materials Science and Engineering, University of Michigan, \\ Ann Arbor, Michigan 48109
}

(Received 10 March 2003; accepted 8 July 2003)

\begin{abstract}
Relaxation in supercooled liquids above their glass transition and below the onset temperature of "slow" dynamics involves the correlated motion of neighboring particles. This correlated motion results in the appearance of spatially heterogeneous dynamics or "dynamical heterogeneity." Traditional two-point time-dependent density correlation functions, while providing information about the transient "caging" of particles on cooling, are unable to provide sufficiently detailed information about correlated motion and dynamical heterogeneity. Here, we study a four-point, time-dependent density correlation function $g_{4}(r, t)$ and corresponding "structure factor" $S_{4}(q, t)$ which measure the spatial correlations between the local liquid density at two points in space, each at two different times, and so are sensitive to dynamical heterogeneity. We study $g_{4}(r, t)$ and $S_{4}(q, t)$ via molecular dynamics simulations of a binary Lennard-Jones mixture approaching the mode coupling temperature from above. We find that the correlations between particles measured by $g_{4}(r, t)$ and $S_{4}(q, t)$ become increasingly pronounced on cooling. The corresponding dynamical correlation length $\xi_{4}(t)$ extracted from the small- $q$ behavior of $S_{4}(q, t)$ provides an estimate of the range of correlated particle motion. We find that $\xi_{4}(t)$ has a maximum as a function of time $t$, and that the value of the maximum of $\xi_{4}(t)$ increases steadily from less than one particle diameter to a value exceeding nine particle diameters in the temperature range approaching the mode coupling temperature from above. At the maximum, $\xi_{4}(t)$ and the $\alpha$ relaxation time $\tau_{\alpha}$ are related by a power law. We also examine the individual contributions to $g_{4}(r, t), S_{4}(q, t)$, and $\xi_{4}(t)$, as well as the corresponding order parameter $Q(t)$ and generalized susceptibility $\chi_{4}(t)$, arising from the self and distinct contributions to $Q(t)$. These contributions elucidate key differences between domains of localized and delocalized particles. (C) 2003 American Institute of Physics.
\end{abstract}

[DOI: $10.1063 / 1.1605094]$

\section{INTRODUCTION}

Understanding the underlying principles of the glass transition is a long-standing problem in condensed matter. Some have argued that the glass transition is a purely kinetic phenomenon, ${ }^{1,2}$ while others propose that the experimentally observed transition is a "ghost" of an underlying thermodynamic transition. ${ }^{3,4}$ Many ideas relating the glass transition to thermodynamics date back to Kauzmann, ${ }^{5}$ who envisioned a relation between the glass transition temperature $\left(T_{g}\right)$ and the entropy difference between the supercooled liquid and stable crystal. Adam, Gibbs, and DiMarzio later expanded on these ideas, ${ }^{6,7}$ proposing that relaxation of the liquid occurs via "cooperatively-rearranging regions," and related dynamical properties to a configurational entropy, later interpreted as a measure of the number of mechanically stable configurations accessible to the liquid as described by
Goldstein, ${ }^{8}$ Stillinger, ${ }^{9}$ and others. ${ }^{10}$ Increased computational power has aided the identification of cooperative motion in supercooled liquids ${ }^{11-17}$ and tests of configurational entropy theories. ${ }^{18-20}$ Simulations ${ }^{11-17}$ and experiments ${ }^{21-36}$ have demonstrated the cooperative and spatially heterogeneous nature of the liquid dynamics (for reviews of the experimental evidence for spatially heterogeneous dynamics, see, e.g., Refs. 37-39). Experimental efforts have been made to measure the length scale of cooperative motion in supercooled liquids using a variety of techniques (see, e.g., Refs. 29, 35, and 40). However, a clear, definitive measure of this length, and its temperature dependence on approaching the glass transition, has been elusive. The presence of a growing correlation length approaching the glass transition might help to motivate using the theoretical framework of conventional critical phenomena, suitably adapted to this problem, to un- 
derstand the glass transition. Indeed, the mode-coupling theory (MCT) (Ref. 41) already incorporates many ideas of critical phenomena, without explicit inclusion of a growing length - either static or dynamic — and has been widely used to describe the slowing down of the dynamics on weak supercooling. This regime corresponds to the $T$ range of our simulations, over which the diffusion constant decreases by 4-6 orders of magnitude from a typical high temperature liquid value of $10^{-5} \mathrm{~cm}^{2} / \mathrm{s}$.

Using the framework of conventional critical phenomena, researchers have searched for a growing, static correlation length that might be associated with the slowing of the dynamics on cooling near the glass transition. However, no evidence for such a length has been found from static twopoint density fluctuations and other traditional correlation functions that measure local static structure. ${ }^{34,42}$ Some success has been obtained using computer simulations by focusing instead on a dynamical correlation length that measures the typical size of dynamically correlated regions, and thus essentially characterizes the distance over which particle motion is correlated. ${ }^{13-17,43-52}$

For example, Onuki and Yamamoto ${ }^{48}$ calculated dynamical correlation length of clusters formed by particles "connected" by bonds that have a length of the order of a size of a particle. Introducing a concept of a bond breakage time (time for particle to move greater then one interparticle distance), and monitoring the bond breakage process, they show that particles with "broken bonds" form clusters. They state that "the heterogeneity structure in the local mobility is very analogous to the critical fluctuations in Ising spin systems." Based on this analogy, they fit the structure factor of the particles pairs with broken bonds to an Orstein-Zernike form (Lorentzian), and found a correlation length that grows as $T$ decreases. Hurley and Harrowell ${ }^{49}$ estimated the "characteristic linear dimension of the kinetic heterogeneities" of a one-component 2D system of soft disks from the second moment of the mean relaxation times of subregions. They found that the characteristic correlation length of these subregions is increasing function of density. Doliwa and Heuer ${ }^{50}$ analyzed displacement correlations in both 2D disks and 3D hards spheres. They were able to measure the "total reduction of degree of freedom and the spatial extent of correlations" of particles. They found that the dynamical correlation length based on single particles displacements during some observation time is increasing function of density, but at times longer than the relaxation time of the system, they found that this correlation length is monotonically increasing function of time with some limiting value. Mountain ${ }^{51}$ calculated a "hydrodynamic length" in MD simulation via a transverse current correlation function corresponding to the longest wavelength of propagating shear waves supported by the liquid, and found to grow with decreasing $T$. In the context of glassforming liquids in confined geometry, Scheindler et $a .^{52}$ used MD simulations to investigate how boundary effects influence the relaxation dynamics and any concomitant effect on the correlation length. They found that the characteristic length scale can be estimated from the "correction" in the intermediate scattering function in the vicinity of the boundary. This characteristic length is also found to grow as $T$ decreases. Below $T_{g}$, Parisi also found an increasing correlation length corresponding to cooperative motion. ${ }^{53}$ Recently, a new theoretical approach to the problem of cooperativity demonstrated how spatially heterogeneous dynamics (SHD) can arise in simple systems with cooperative dynamics. ${ }^{55}$ This theory predicts a growing correlation length on decreasing $T .{ }^{54}$ In Refs. 17, 43-47, and 56, several approaches-including calculation of a displacementdisplacement correlation function and identification of clusters of mobile particles-predicted that the characteristic length scale of dynamically similar regions grows on cooling, while the static correlation length remains nearly unchanged. Many specific predictions made possible through such analysis have now been confirmed in experiments on colloidal suspensions. ${ }^{32,33,57}$

The temporary localization or "caging" of particles by their neighbors-which is associated with dynamical heterogeneity-is readily observable from probing the timedependence of density fluctuations via scattering methods. However, direct measurement of a length scale via scattering experiments characterizing correlated particle motion in liquids is more complicated, because this length involves the motion of two or more particles, and hence a four-point function containing information about the density at two points, each at two times, is required. Traditional two-point, timedependent density correlation functions (such as the intermediate scattering function) depend on at most the density at two different locations, but each at only one time.

Dasgupta et $a .^{58}$ were the first to propose the use of a four-point, time-dependent density correlation function $g_{4}(r, t)$ in simulations of supercooled liquids. However, they did not find evidence of an increasing length as the temperature is decreased. More recently, this function was revisited and the theoretical framework expanded by Refs. 59 and 60, who focused on a four-point, time-dependent susceptibility given by the volume integral of $g_{4}(r, t)$. Using both theoretical calculations ${ }^{59}$ and simulation, ${ }^{60}$ these authors argued for the existence of a growing correlation length on cooling based on the increase of the dynamical susceptibility with decreasing temperature. Compared with previous studies of SHD, that work provides a rigorous exposition of a theoretical framework within which to relate SHD to the traditional correlation functions used to describe the liquid state. The function $g_{4}(r, t)$ is consequently important within the context of liquid state theory, and this is our primary motivation for its thorough investigation, as presented in this paper.

The principal goal of this paper is to further develop the formalism for $g_{4}(r, t)$, calculate $g_{4}(r, t)$ via molecular dynamics simulations of a model glassforming liquid, and quantify the temperature dependence of its characteristic length scale $\xi_{4}(t)$. Additionally, we explore the individual contributions to the correlation function from particles that are either temporarily immobile (localized) or mobile, and elucidate the structure of domains of localized particles, which were shown to dominate the correlation function in Ref. 60. A preliminary account of this work appears in Ref. 61. We note a key difference between the results we reported 
TABLE I. Average temperature $\langle T\rangle$, kinetic and potential energy per particle $\left\langle E_{\text {tot }} / N\right\rangle$, pressure $\langle P\rangle$, relaxation time $\tau_{\alpha}$, equilibration time $t_{\mathrm{eq}}$ since previous run, and production run time $t_{\mathrm{pr}}$ for each state point simulated. The density for each state point is $\rho=1.296$.

\begin{tabular}{cccccc}
\hline \hline$\langle T\rangle$ & $\left\langle E_{\text {tot }} / N\right\rangle$ & $\langle P\rangle$ & $\tau_{\alpha}$ & $t_{\mathrm{eq}}$ & $t_{\mathrm{pr}}$ \\
\hline $0.588 \pm 0.001$ & $-5.1900 \pm 0.0004$ & $6.479 \pm 0.001$ & $3500 \pm 100$ & $6.9 \times 10^{5}$ & $7.8 \times 10^{5}$ \\
$0.598 \pm 0.002$ & $-5.1507 \pm 0.0004$ & $6.660 \pm 0.002$ & $1900 \pm 100$ & $4.6 \times 10^{5}$ & $7.8 \times 10^{5}$ \\
$0.615 \pm 0.001$ & $-5.0790 \pm 0.0002$ & $6.976 \pm 0.001$ & $880 \pm 50$ & $3.4 \times 10^{5}$ & $4.6 \times 10^{5}$ \\
$0.637 \pm 0.001$ & $-4.9922 \pm 0.0002$ & $7.359 \pm 0.001$ & $370 \pm 50$ & $1.1 \times 10^{5}$ & $1.1 \times 10^{5}$ \\
$0.660 \pm 0.001$ & $-4.9008 \pm 0.0002$ & $7.755 \pm 0.003$ & $240 \pm 30$ & $4.6 \times 10^{4}$ & $1.1 \times 10^{5}$ \\
$0.689 \pm 0.001$ & $-4.8009 \pm 0.0002$ & $8.178 \pm 0.001$ & $150 \pm 30$ & $2.3 \times 10^{4}$ & $1.1 \times 10^{5}$ \\
$0.944 \pm 0.001$ & $-3.9508 \pm 0.0002$ & $11.603 \pm 0.002$ & $16 \pm 5$ & $2.3 \times 10^{4}$ & $1.1 \times 10^{5}$ \\
$2.004 \pm 0.001$ & $-0.8981 \pm 0.0002$ & $22.541 \pm 0.002$ & $4 \pm 1$ & $1.1 \times 10^{4}$ & $1.1 \times 10^{5}$ \\
\hline \hline
\end{tabular}

in Ref. 61 and those we report here. In the present paper, we estimate the correlation length corresponding to a correlation function $g_{4}^{o l}(r, t)$, which is related to the correlation function $g_{4}^{*}(r, t)$ investigated in Ref. 61, using a wider range of data and different approach. We now estimate a correlation length exceeding nine particle diameters within $5 \%$ of $T_{\text {MCT }}$.

The paper is organized as follows: In Sec. II we describe the model and simulation details. In Sec. III we review and extend the theoretical treatment of Refs. 59-61 to rewrite the four-point density correlation function in a manner suitable for calculation. In Sec. IV we present numerical results for the order parameter, susceptibility, four-point correlation function, four-point structure factor and dynamical correlation length. We conclude with a discussion of our results in Sec. V.

\section{MODEL AND SIMULATION DETAILS}

We study a 50/50 binary mixture of particle types " $A$ " and " $B$ " which interact via the Lennard-Jones potential,

$$
V_{\alpha \beta}(r)=4 \epsilon_{\alpha \beta}\left[\left(\frac{\sigma_{\alpha \beta}}{r}\right)^{12}-\left(\frac{\sigma_{\alpha \beta}}{r}\right)^{6}\right] .
$$

This system has been studied previously by Wahnstrom ${ }^{62}$ and Schrøder. ${ }^{63}$ Following these authors, we use length parameters $\sigma_{A A}=1, \sigma_{B B}=5 / 6$, and $\sigma_{A B}=\left(\sigma_{A A}+\sigma_{B B}\right) / 2$, and energy parameters $\epsilon_{A A}=\epsilon_{B B}=\epsilon_{A B}=1$. The masses of the particles are chosen to be $m_{A}=2$ and $m_{B}=1$. We shift the potential and truncate it so it vanishes at $r=2.5 \sigma_{A B}$.

We simulate a system of $N=8000$ particles using periodic boundary conditions in a cubic box of length $L=18.344$ in units of $\sigma_{A A}$, which yields a density of $\rho=N / L^{3}=1.296$ for all state points. We report time in units of $\tau=\left(m_{B} \sigma_{A A}^{2} / 48 \epsilon_{A A}\right)^{\frac{1}{2}}$, length in units of $\sigma_{A A}$, and temperature $T$ in units of $\epsilon_{A A} / k$, where $k$ is Boltzmann's constant. The simulations are performed using LAMMPS, a publicly available parallel MD simulation code developed by Plimpton. ${ }^{64}$ We simulate eight state points at temperatures ranging from $T=2.0$ to $T=0.59$, following a constant volume path similar to that followed in Refs. 60, 63, 65, and 66. The simulations are performed in the NVE ensemble. Each state point is generated from an equilibrated state point at higher temperature. We first perform an equilibration run of length $t_{\mathrm{eq}}$, to allow the kinetic and potential energies to relax from their values at the previous state point to their equilib- rium values at the new state point. The length of $t_{\mathrm{eq}}$ for each temperature is several multiples of the time needed for the equilibrium coherent intermediate scattering function,

$$
F\left(\mathbf{q}_{0}, t\right)=\frac{\left\langle\Sigma_{\mathrm{jk}} \exp \left[i \mathbf{q}_{0} \cdot \mathbf{r}_{j}(0)\right] \exp \left[i \mathbf{q}_{0} \cdot \mathbf{r}_{k}(t)\right]\right\rangle}{\left\langle\sum_{\mathrm{jk}} \exp \left[i \mathbf{q}_{0} \cdot \mathbf{r}_{j}(0)\right] \exp \left[i \mathbf{q}_{0} \cdot \mathbf{r}_{k}(0)\right]\right\rangle}
$$

(Ref. 67), to decay to zero, where $q_{0}=\left|\mathbf{q}_{0}\right| \approx 7.5$ corresponds to the maximum of the static structure factor. To ensure that the systems are equilibrated prior to data collection, we also check that there is no aging in dynamical quantities such as the mean square displacement, and ensure that all properties of interest are independent of time origin. Once equilibrium is established, we perform a production run of length $t_{\mathrm{pr}}$ $=b \tau_{\alpha}$, where $\tau_{\alpha}$ is the $T$-dependent relaxation time of $F\left(q_{0}, t\right)$, and where $b \approx 10^{3}$ for the highest $T$ and $b \approx 200$ for the lowest $T$ studied. We calculate $\tau_{\alpha}$ by fitting the secondary relaxation of $F\left(q_{0}, t\right)$ to a stretched exponential function $F(t)=A \exp \left(-\left(t / \tau_{\alpha}\right)^{\beta}\right)$. We perform the fitting for $t>0.6$ for the two highest temperatures and $t>20$ for the rest of the temperatures. During the production run, we save the particle positions at logarithmically spaced time intervals for later analysis. We summarize the equilibrium thermodynamic properties, relaxation time $\tau_{\alpha}$, equilibration time $t_{\mathrm{eq}}$, and production run time $t_{\mathrm{pr}}$ for all state points studied in Table I. The calculation of error bars is explained in Ref. 68 .

The bulk pair correlation function

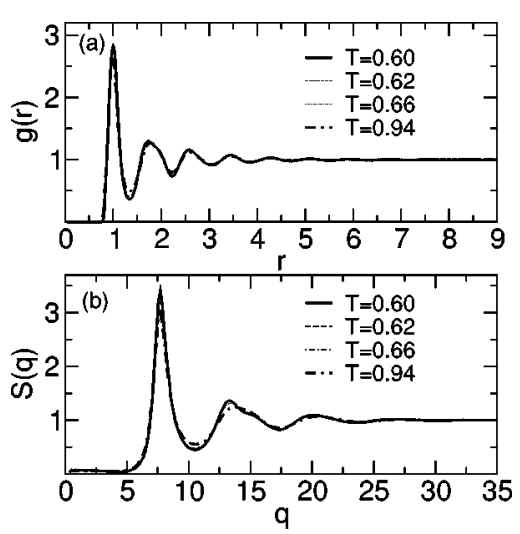

FIG. 1. (a) $T$-dependence of pair correlation function $g(r)$. (b) $T$-dependence of static structure factor $S(q)$. 


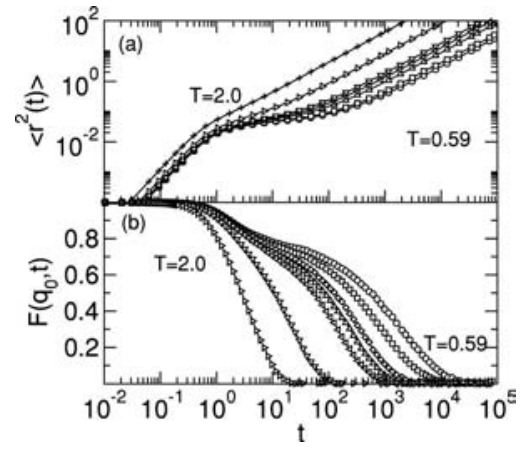

FIG. 2. (a) Mean-square displacement for several state points studied. (b) Coherent intermediate scattering function $F\left(q_{0}, t\right)$ calculated at $q_{0} \approx 7.5$ for several state points studied.

$$
g(\mathbf{r})=\frac{V}{N^{2}} \sum_{i \neq j} \delta\left(\mathbf{r}-\mathbf{r}_{i}+\mathbf{r}_{j}\right)
$$

and its related Fourier transform, the static structure factor

$$
S(\mathbf{q})=\frac{1}{N}\langle\rho(\mathbf{q}) \rho(-\mathbf{q})\rangle,
$$

where $\rho(\mathbf{q})=\Sigma_{j} \exp \left[i \mathbf{q} \cdot \mathbf{r}_{j}\right],{ }^{67}$ are shown in Fig. 1. As observed in many other supercooled liquids, these functions exhibit only a very weak $T$-dependence. In contrast, the mean square displacement $\left\langle r^{2}(t)\right\rangle$ and coherent intermediate scattering function $F\left(q_{0}, t\right)$ at the peak wave vector are shown in Fig. 2; they display qualitatively the same $T$-dependence as other well-studied glass-forming liquids.

We estimate the mode coupling temperature $T_{\mathrm{MCT}}$ from a power law fit, ${ }^{41}$

$$
\tau_{\alpha}(T) \sim\left(T / T_{\mathrm{MCT}}-1\right)^{-\gamma}
$$

of the $T$-dependence of $\tau_{\alpha}$ and find $T_{\mathrm{MCT}}=0.57 \pm 0.01$. The value of $T_{\mathrm{MCT}}$ obtained from fits of the diffusion constant $D$ is slightly higher: ${ }^{63} T_{\mathrm{MCT}}=0.57 \pm 0.01$. We estimate the Kauzmann temperature $T_{0}$, which can be considered a lower bound for the glass transition temperature $T_{g}$, from a fit using the Vogel-Fulcher-Tammann (VFT) form,

$$
\tau_{\alpha}(T) \sim \exp \left(A /\left(T / T_{0}-1\right)\right),
$$

where $A$ is the activation energy. The data and fit are shown in Fig. 7; we find $T_{0}=0.48 \pm 0.02$. The error bars are confidence intervals obtained as a result of fitting $\tau_{\alpha}(T)$ to a power law and exponential form, respectively. The onset of "supercooled" liquid behavior, as indicated by the appearance of a plateau in the mean square displacement, is observed to occur at $T \approx 1.0$, as reported in Ref. 65 .

\section{THEORY}

\section{A. General framework}

We first review the general framework of the four-point, time-dependent, density correlation function and related quantities, previously presented in briefer form in Refs. 5961 , and extended here. Consider a liquid of $N$ particles occupying a volume $V$, with density

$$
\rho(\mathbf{r}, t)=\sum_{i=1}^{N} \delta\left(\mathbf{r}-\mathbf{r}_{i}(t)\right)
$$

A time-dependent order parameter may be defined in terms of the function,

$$
\begin{aligned}
Q_{p}(t) & \equiv \int d \mathbf{r}_{1} d \mathbf{r}_{2} \rho\left(\mathbf{r}_{1}, 0\right) \rho\left(\mathbf{r}_{2}, t\right) \delta\left(\mathbf{r}_{1}-\mathbf{r}_{2}\right) \\
& =\sum_{i=1}^{N} \sum_{j=1}^{N} \delta\left(\mathbf{r}_{i}(0)-\mathbf{r}_{j}(t)\right),
\end{aligned}
$$

which measures the number of "overlapping" particles in two configurations separated by a time interval $t$. The fluctuations in a time-dependent order parameter $\chi_{4}^{p}(t)$ may be defined as

$$
\chi_{4}^{p}(t)=\frac{\beta V}{N^{2}}\left[\left\langle Q_{p}^{2}(t)\right\rangle-\left\langle Q_{p}(t)\right\rangle^{2}\right] .
$$

Additionally, we may write

$$
\chi_{4}^{p}(t) \equiv \frac{\beta V}{N^{2}} \int d \mathbf{r}_{1} d \mathbf{r}_{2} d \mathbf{r}_{3} d \mathbf{r}_{4} \mathcal{G}_{4}\left(\mathbf{r}_{1}, \mathbf{r}_{2}, \mathbf{r}_{3}, \mathbf{r}_{4}, t\right),
$$

where $\beta=\left(k_{B} T\right)^{-1}$, and

$$
\begin{aligned}
& \mathcal{G}_{4}\left(\mathbf{r}_{1}, \mathbf{r}_{2}, \mathbf{r}_{3}, \mathbf{r}_{4}, t\right) \\
& \equiv\left\langle\rho\left(\mathbf{r}_{1}, 0\right) \rho\left(\mathbf{r}_{2}, t\right) \delta\left(\mathbf{r}_{1}-\mathbf{r}_{2}\right) \rho\left(\mathbf{r}_{3}, 0\right) \rho\left(\mathbf{r}_{4}, t\right) \delta\left(\mathbf{r}_{3}-\mathbf{r}_{4}\right)\right\rangle \\
&-\left\langle\rho\left(\mathbf{r}_{1}, 0\right) \rho\left(\mathbf{r}_{2}, t\right) \delta\left(\mathbf{r}_{1}-\mathbf{r}_{2}\right)\right\rangle \\
& \times\left\langle\rho\left(\mathbf{r}_{3}, 0\right) \rho\left(\mathbf{r}_{4}, t\right) \delta\left(\mathbf{r}_{3}-\mathbf{r}_{4}\right)\right\rangle,
\end{aligned}
$$

is a four-point, time-dependent, density correlation function. The form of Eq. (7) is analogous to the isothermal compressibility $\kappa_{T}$, which is proportional to the volume integral of the static density correlation function $g(\mathbf{r})-1 .^{67}$

Note that $\left\langle Q_{p}(t)\right\rangle=N G(0, t)$ where $G(r, t)$ is the timedependent, van Hove two-point density correlation function. Thus $\left\langle Q_{p}(t)\right\rangle$ quickly decays to the long time limit $\lim _{t \rightarrow \infty}\left\langle Q_{p}(t)\right\rangle=1 / V$ due to the vibrational motion of the particles. ${ }^{67}$ In this paper, we are interested in probing the correlated motion of particles in liquids approaching the glass transition. It has been demonstrated in several papers ${ }^{17,43-45,69}$ that vibrational motion is only weakly correlated at best; strong correlations appear only on longer time scales when particles move a substantial distance and escape from their cages. To capture this motion using the four-point correlation function of Eq. (8), Refs. 59, 60, and 70 introduced a "coarse-graining" by defining a counterpart $Q(t)$ to $Q_{p}(t)$ in terms of a parameter $a$, associated with the typical amplitude of the vibrational motion of the particles. Following these authors, we modify $Q_{p}(t)$ by an "overlap" function $w\left(\left|\mathbf{r}_{1}-\mathbf{r}_{2}\right|\right)$ that is unity for $\left|\mathbf{r}_{1}-\mathbf{r}_{2}\right| \leqslant a$ and zero otherwise. Equation (5) becomes

$$
\begin{aligned}
Q(t) & =\int d \mathbf{r}_{1} \mathbf{r}_{2} \rho\left(\mathbf{r}_{1}, 0\right) \rho\left(\mathbf{r}_{2}, t\right) w\left(\left|\mathbf{r}_{1}-\mathbf{r}_{2}\right|\right) \\
& =\sum_{i=1}^{N} \sum_{j=1}^{N} w\left(\left|\mathbf{r}_{i}(0)-\mathbf{r}_{j}(t)\right|\right)
\end{aligned}
$$


Physically, $Q(t)$ is the overlap between a configuration of the system at a reference time $t=0$ and at a later time $t$; that is, $Q(t)$ is equal to the number of particles that in a time $t$ either remained within a distance $a$ of their original position, or were replaced by another particle. Note that the long time limit $Q_{\infty} \equiv \lim _{t \rightarrow \infty} Q(t) \neq 1 / V$ because of the nonzero random probability of finding overlapping particles. $Q_{\infty} / N$ is given by the probability of a "random overlap," i.e., the fraction of the volume occupied by particles at any given time, $N V_{a} / V=\rho V_{a}$, where $V_{a}=4 / 3 \pi a^{3}$.

Replacing $Q(t)$ in Eq. (6) yields finally a modified expression for the susceptibility,

$$
\chi_{4}(t)=\frac{\beta V}{N^{2}}\left[\left\langle Q(t)^{2}\right\rangle-\langle Q(t)\rangle^{2}\right] .
$$

Expressing $\chi_{4}(t)$ in terms of the four-point correlation function $G_{4}\left(\mathbf{r}_{1}, \mathbf{r}_{2}, \mathbf{r}_{3}, \mathbf{r}_{4}, t\right)$, we obtain

$$
\chi_{4}(t)=\frac{\beta V}{N^{2}} \int d \mathbf{r}_{1} d \mathbf{r}_{2} d \mathbf{r}_{3} d \mathbf{r}_{4} G_{4}\left(\mathbf{r}_{1}, \mathbf{r}_{2}, \mathbf{r}_{3}, \mathbf{r}_{4}, t\right),
$$

where

$$
\begin{aligned}
G_{4}\left(\mathbf{r}_{1}, \mathbf{r}_{2}, \mathbf{r}_{3}, \mathbf{r}_{4}, t\right)= & \left\langle\rho\left(\mathbf{r}_{1}, 0\right) \rho\left(\mathbf{r}_{2}, t\right) w\left(\left|\mathbf{r}_{1}-\mathbf{r}_{2}\right|\right)\right. \\
& \left.\times \rho\left(\mathbf{r}_{3}, 0\right) \rho\left(\mathbf{r}_{4}, t\right) w\left(\left|\mathbf{r}_{3}-\mathbf{r}_{4}\right|\right)\right\rangle \\
& -\left\langle\rho\left(\mathbf{r}_{1}, 0\right) \rho\left(\mathbf{r}_{2}, t\right) w\left(\left|\mathbf{r}_{1}-\mathbf{r}_{2}\right|\right)\right\rangle \\
& \times\left\langle\rho\left(\mathbf{r}_{3}, 0\right) \rho\left(\mathbf{r}_{4}, t\right) w\left(\left|\mathbf{r}_{3}-\mathbf{r}_{4}\right|\right)\right\rangle .
\end{aligned}
$$

Note that Eq. (12) collapses to Eq. (8) if each $w\left(\left|\mathbf{r}_{i}-\mathbf{r}_{j}\right|\right)$ is replaced by $\delta\left(\left|\mathbf{r}_{i}-\mathbf{r}_{j}\right|\right)$.

In the case of both the mean-field, $p$-spin model and a liquid in the hypernetted chain approximation, ${ }^{71-74}$ the time dependence of $\chi_{4}(t)$ was calculated numerically from an analytic expression in Ref. 59. Reference 59 showed that $\chi_{4}(t)$ is maximum at some intermediate time $t_{4}^{\max }$, and both the position and the height of this maximum increase as a power law as $T \rightarrow T_{\mathrm{MCT}}^{+}$. Those calculations provide the first analytical prediction of the growth of a generalized dynamical susceptibility and, by inference, a corresponding dynamical correlation length $\xi_{4}(t)$ in a model glass-forming system. We will calculate this length later in this chapter.

\section{B. Derivation of radially-averaged four-point correlation function $g_{4}(r, t)$}

We wish to radially average the four-point correlation function in Eq. (12) to obtain a function $g_{4}(r, t)$ that depends only on the magnitude $r$ of the distance between two particles at time $t=0$. We start from the requirement that

$$
\chi_{4}(t)=\beta \int d \mathbf{r} g_{4}(\mathbf{r}, t)
$$

To achieve this, we first integrate over $\mathbf{r}_{2}$ and $\mathbf{r}_{4}$ in Eq. (11) and obtain

$$
\begin{aligned}
\chi_{4}(t)= & \frac{\beta V}{N^{2}} \int d \mathbf{r}_{1} d \mathbf{r}_{3} \sum_{i j k l}\left[\left\langle\delta\left(\mathbf{r}_{1}-\mathbf{r}_{i}(0)\right)\right.\right. \\
& \left.\times \delta\left(\mathbf{r}_{3}-\mathbf{r}_{k}(0)\right) w\left(\left|\mathbf{r}_{1}-\mathbf{r}_{j}(t)\right|\right) w\left(\left|\mathbf{r}_{3}-\mathbf{r}_{l}(t)\right|\right)\right\rangle \\
& -\left\langle\delta\left(\mathbf{r}_{1}-\mathbf{r}_{i}(0)\right) w\left(\left|\mathbf{r}_{1}-\mathbf{r}_{j}(t)\right|\right)\right\rangle \\
& \left.\times\left\langle\delta\left(\mathbf{r}_{3}-\mathbf{r}_{k}(0)\right) w\left(\left|\mathbf{r}_{3}-\mathbf{r}_{l}(t)\right|\right)\right\rangle\right] .
\end{aligned}
$$

Defining $\mathbf{r}$ as $\mathbf{r}=\mathbf{r}_{3}-\mathbf{r}_{1}$ and integrating over $\mathbf{r}_{3}$ in Eq. (14) we find

$$
\begin{aligned}
\chi_{4}(t)= & \frac{\beta V}{N^{2}} \sum_{i j k l}\left[\int d \mathbf { r } \left\langle\delta\left(\mathbf{r}-\mathbf{r}_{k}(0)+\mathbf{r}_{i}(0)\right) w\left(\left|\mathbf{r}_{i}(0)-\mathbf{r}_{j}(t)\right|\right)\right.\right. \\
& \left.\times w\left(\left|\mathbf{r}_{k}(0)-\mathbf{r}_{l}(t)\right|\right)\right\rangle-\left\langle w\left(\left|\mathbf{r}_{i}(0)-\mathbf{r}_{j}(t)\right|\right)\right\rangle \\
& \left.\times\left\langle w\left(\left|\mathbf{r}_{k}(0)-\mathbf{r}_{l}(t)\right|\right)\right\rangle\right] .
\end{aligned}
$$

Choosing to integrate over different variables would result in a function that calculates spatial correlations between overlapping particles at either time $t$ or at a combination of times. This choice makes little qualitative difference to our results if $a$ is small, as it is in our calculations (see Appendix).

Combining Eqs. (9), (13), and (15), we obtain $g_{4}(\mathbf{r}, t)$,

$$
\begin{aligned}
g_{4}(\mathbf{r}, t)= & \frac{1}{N \rho}\left\langle\sum_{i j k l} \delta\left(\mathbf{r}-\mathbf{r}_{k}(0)+\mathbf{r}_{i}(0)\right) w\left(\left|\mathbf{r}_{i}(0)-\mathbf{r}_{j}(t)\right|\right)\right. \\
& \left.\times w\left(\left|\mathbf{r}_{k}(0)-\mathbf{r}_{l}(t)\right|\right)\right\rangle-\left\langle\frac{Q(t)}{N}\right\rangle^{2}
\end{aligned}
$$

We investigate the behavior of $g_{4}(r, t)$, which is the radially averaged function of a single variable $r$. Assuming an isotropic system, $g_{4}(r, t)$ is a function of only the magnitude $r=|\mathbf{r}|$. With the above choice of integration variables, $g_{4}(r, t)$ describes spatial correlations between overlapping particles separated by a distance $r$ at the initial time (using information at time $t$ to label the overlapping particles). The first term in $g_{4}(r, t)$ is a pair correlation function restricted to the subset of overlapping particles. We define this pair correlation function of overlapping particles as $g_{4}^{o l}(r, t)$. The second term represents the probability of any two randomly chosen particles overlapping at times 0 and $t$. We can thus define $g_{4}(r, t)$ as

$$
g_{4}(r, t) \equiv g_{4}^{o l}(r, t)-\left\langle\frac{Q(t)}{N}\right\rangle^{2} .
$$

By factoring out the quantity $\langle Q(t) / N\rangle^{2}, g_{4}(r, t)$ can also be written in the form,

$$
\begin{aligned}
g_{4}(r, t) & =\left\langle\frac{Q(t)}{N}\right\rangle^{2}\left[\frac{g_{4}^{o l}(r, t)}{\left\langle\frac{Q(t)}{N}\right\rangle^{2}}-1\right] \\
& \equiv\left\langle\frac{Q(t)}{N}\right\rangle^{2} g_{4}^{*}(r, t) .
\end{aligned}
$$

Written this way, the function $g_{4}^{*}(r, t)=g(r)-1$ at $t=0$, and $g_{4}^{*}(r, t)=0$ in the absence of correlations. We studied the behavior of $g_{4}^{*}(r, t)$ in Ref. 61. In this paper we study the behavior of $g_{4}^{o l}(r, t)$, using a different approach to calculate correlation length that provides better estimate than that ob 
tained in Ref. 61. The correlation length of each of these functions must be the same.

\section{Four-point, time-dependent structure factor $S_{4}(q, t)$}

The structure factor that corresponds to $g_{4}(\mathbf{r}, t)$ is its Fourier transform,

$$
\begin{aligned}
S_{4}(\mathbf{q}, r)= & \int g_{4}(\mathbf{r}, t) \exp [-i \mathbf{q} \cdot \mathbf{r}] d \mathbf{r} \\
= & \int g_{4}^{o l}(\mathbf{r}, t) \exp [-i \mathbf{q} \cdot \mathbf{r}] d \mathbf{r}-\left\langle\frac{Q(t)}{N}\right\rangle^{2} \\
& \times \int \exp [-i \mathbf{q} \cdot \mathbf{r}] d \mathbf{r} .
\end{aligned}
$$

Equation (19) implies that

$$
\lim _{q \rightarrow 0} S_{4}(q, t)=\frac{\chi_{4}(t)}{\beta} .
$$

Since the second term in Eq. (19) is proportional to $\delta(\mathbf{q})$, we are only interested in the first term of Eq. (19). We define a four-point, time-dependent structure factor of overlapping particles $S_{4}^{o l}(\mathbf{q}, t)$ as the Fourier transform of $g_{4}^{o l}(r, t)$,

$$
\begin{aligned}
S_{4}^{o l}(\mathbf{q}, t)= & \int g_{4}^{o l}(\mathbf{r}, t) \exp [-i \mathbf{q} \cdot \mathbf{r}] d \mathbf{r} \\
= & \int d \mathbf{r} \exp [-i \mathbf{q} \cdot \mathbf{r}]\left\langle\frac{1}{N \rho} \sum_{i j k l} \delta\left(\mathbf{r}-\mathbf{r}_{k}(0)+\mathbf{r}_{i}(0)\right)\right. \\
& \left.\times w\left(\left|\mathbf{r}_{i}(0)-\mathbf{r}_{j}(t)\right|\right) w\left(\left|\mathbf{r}_{k}(0)-\mathbf{r}_{l}(t)\right|\right)\right\rangle \\
= & \left\langle\frac{1}{N \rho} \sum_{i j k l} \exp \left[-i \mathbf{q} \cdot\left(\mathbf{r}_{k}(0)-\mathbf{r}_{i}(0)\right)\right]\right. \\
& \left.\times w\left(\left|\mathbf{r}_{i}(0)-\mathbf{r}_{j}(t)\right|\right) w\left(\left|\mathbf{r}_{k}(0)-\mathbf{r}_{l}(t)\right|\right)\right\rangle \\
= & \frac{1}{N \rho}\left\langle\sum_{i j} \exp \left[i \mathbf{q} \cdot \mathbf{r}_{i}(0)\right] w\left(\left|\mathbf{r}_{i}(0)-\mathbf{r}_{j}(t)\right|\right)\right. \\
& \left.\times \sum_{k l} \exp \left[-i \mathbf{q} \cdot \mathbf{r}_{k}(0)\right] w\left(\left|\mathbf{r}_{k}(0)-\mathbf{r}_{l}(t)\right|\right)\right\rangle
\end{aligned}
$$

To write Eq. (21) in a more compact form, we define

$$
\rho^{o l}(\mathbf{q}, t)=\sum_{i j} \exp \left[i \mathbf{q} \cdot \mathbf{r}_{i}(0)\right] w\left(\left|\mathbf{r}_{i}(0)-\mathbf{r}_{j}(t)\right|\right),
$$

and obtain

$$
S_{4}^{o l}(\mathbf{q}, t)=\frac{1}{N \rho}\left\langle\rho^{o l}(\mathbf{q}, t) \rho^{o l}(-\mathbf{q}, t)\right\rangle .
$$

Equation (21) is analogous to the static structure factor $S(\mathbf{q})$, but "scatters" off of overlapping particles using information on overlapping particles at time $t$ to label particles at time 0 . In Eq. (21), $\rho=N / V=1.296$ is the average density in the system defined in Sec. II. In the following, we calculate the angularly averaged $S_{4}^{o l}(q, t)$.

\section{Self and distinct contributions to $Q(t), \chi_{4}(t)$, $g_{4}(r, t)$, and $S_{4}^{o l}(q, t)$}

The contribution of a given particle $i$ to $Q(t)$ is a result of three possible events: (i) particle $i$ remains within a distance $a$ of its original position; (ii) particle $i$ moves and is replaced (within a distance $a$ ) by another particle; or (iii) particle $i$ moves a distance greater than $a$ and is not replaced by another particle. Case (iii) does not count as an overlap, and thus does not contribute to $Q(t)$. Cases (i) and (ii) count as overlaps and contribute to the value of $Q(t)$. However, the two cases clearly represent two very different physical situations. To elucidate the various contributions to the fourpoint correlation function, we separate $Q$ into self and distinct components, $Q(t)=Q_{S}(t)+Q_{D}(t)$. The self part,

$$
Q_{S}(t)=\sum_{i=1}^{N} w\left(\left|\mathbf{r}_{i}(t)-\mathbf{r}_{i}(0)\right|\right),
$$

corresponds to terms with $i=j$ in Eq. (9), and measures the number of particles that move less than a distance $a$ in a time interval $t$; we call these "localized" particles. It is tempting to associate localized particles with caged particles, and in many instances this association is valid. However, while a localized particle is always a caged particle, one cannot strictly identify caged particles with localized particles, since a caged particle may occasionally oscillate over a distance larger than $a$ and return to its initial location, and thus would not be considered localized according to the present definition (see Appendix). ${ }^{75}$

The distinct part of $Q(t)$,

$$
Q_{D}(t)=\sum_{i=1}^{N} \sum_{i \neq j}^{N} w\left(\left|\mathbf{r}_{i}(0)-\mathbf{r}_{j}(t)\right|\right),
$$

corresponds to terms with $i \neq j$ in Eq. (9) and gives the number of particles replaced within a radius $a$ by another particle in time $t$.

Following the scheme of decomposing $Q(t), \chi_{4}(t)$ can be decomposed into self $\chi_{S S}(t)$, distinct $\chi_{D D}(t)$, and cross $\chi_{S D}(t)$ terms: $\chi_{4}(t)=\chi_{S S}(t)+\chi_{D D}(t)+\chi_{S D}(t)$, where $\chi_{S S}(t) \propto\left\langle Q_{S}^{2}(t)\right\rangle-\left\langle Q_{S}(t)\right\rangle^{2}, \quad \chi_{D D}(t) \propto\left\langle Q_{D}^{2}(t)\right\rangle-\left\langle Q_{D}(t)\right\rangle^{2}$, and $\chi_{S D}(t) \propto\left\langle Q_{S}(t) Q_{D}(t)\right\rangle-\left\langle Q_{S}(t)\right\rangle\left\langle Q_{D}(t)\right\rangle$. Thus $\chi_{S S}(t)$ is the susceptibility arising from fluctuations in the number of localized particles, $\chi_{D D}(t)$ is the susceptibility arising from fluctuations in the number of particles that are replaced by a neighboring particle, and $\chi_{S D}(t)$ represents cross fluctuations between the number of localized and replaced particles.

We also consider "delocalized" particles, that is, particles that in a time $t$ are more than a distance $a$ from their original location. As was pointed out in Ref. 60, substituting $1-w$ for $w$ in Eq. (24) gives the delocalized order parameter $Q_{D L}(t)=N-Q_{S}(t)$, and as a result, $\chi_{D L}(t)=\chi_{S S}(t)$.

In general, $g_{4}(r, t)$ can be decomposed into more than four terms, depending on the combinations of indices $i, j, k, l$ one considers. In our analysis, we investigate $g_{4}(r, t)$ of localized $\left(g_{4}^{S S}(r, t), i=j\right.$ and $\left.l=k\right)$, replaced $\left(g_{4}^{D D}(r, t), i \neq j\right.$ and $l \neq k)$, localized-replaced $\left(g_{4}^{S D}(r, t), i=j\right.$ and $\left.l \neq k\right)$, and delocalized particles $\left(g_{4}^{D L}(r, t)\right.$, substituting $1-w$ for $w$ and taking $i=j$ and $l=k$ ) in Eq. (16) respectively. Likewise, we 


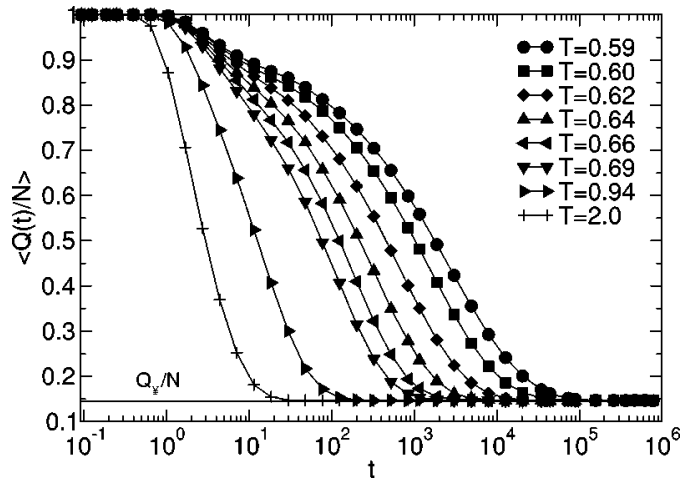

FIG. 3. Average time-dependent overlap "order parameter" $\langle Q(t) / N\rangle$ for each state point simulated. The solid line corresponds to the random value $Q_{\infty} / N=0.147$, as discussed in the previous section.

can decompose $S_{4}^{o l}(q, t)$ into localized $\left(S_{4}^{o l} S S(q, t), i=j\right.$ and $l=k)$, replaced $\left(S_{4}^{o l D D}(q, t), i \neq j\right.$ and $\left.l \neq k\right)$, localizedreplaced $\left(S_{4}^{o l S D}(q, t), \quad i=j\right.$ and $\left.l \neq k\right)$, and delocalized $\left(S_{4}^{o l D L}(q, t)\right.$, substituting $1-w$ for $w$ and taking $i=j$ and $l$ $=k$ ) in Eq. (21), respectively.

Following Eq. (16) and Eq. (17), we write in compact form,

$$
\begin{aligned}
& g_{4}^{S S}(r, t)=\left\langle\frac{Q_{S}(t)}{N}\right\rangle^{2} g_{4}^{S S *}(r, t), \\
& g_{4}^{D D}(r, t)=\left\langle\frac{Q_{D}(t)}{N}\right\rangle^{2} g_{4}^{D D *}(r, t), \\
& g_{4}^{S D}(r, t)=\left\langle\frac{Q_{S}(t)}{N}\right\rangle\left\langle\frac{Q_{D}(t)}{N}\right\rangle g_{4}^{S D *}(r, t),
\end{aligned}
$$

and

$$
g_{4}^{D L}(r, t)=\left\langle\frac{Q_{D L}(t)}{N}\right\rangle^{2} g_{4}^{D L *}(r, t) .
$$

\section{RESULTS}

\section{A. Calculation of $Q(t)$}

We begin by examining the $T$-dependence of the overlap parameter $Q(t)$. We choose $a=0.3$; details about this choice are presented in the Appendix. Figure 3 shows that for all sufficiently low $T, Q(t)$ is characterized by a two-step relaxation, commonly observed in the intermediate scattering function, ${ }^{37}$ as a result of the transient caging of particles. At short times, particles oscillate in a region smaller than the overlap radius $a$, and so $Q / N=1$. We observe a short, initial relaxation of $Q(t)$, and a longer, secondary relaxation.

In Figs. 4(a)-4(c) we show at short, intermediate, and late times $t$, snapshots of overlapping particles; that is, particles that within $t$ are either replaced by another particle or have not moved. At early $t$, few if any particles have moved, and thus the total number of overlaps is close to $N$. At late times, all particles have moved from their initial positions, and only random overlaps remain.

We also consider the individual contributions $Q^{A}(t)$ and $Q^{B}(t)$ to $Q(t)$ from each of the two species of the liquid.
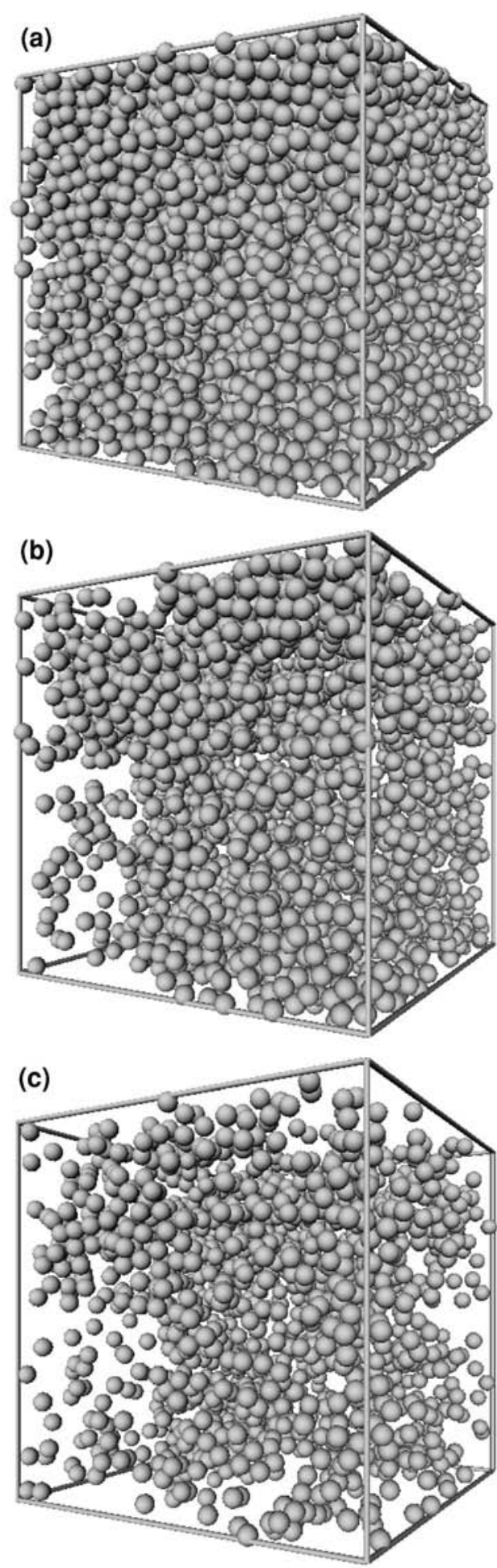

FIG. 4. Snapshot of overlaps at $T=0.60$, at (a) early time, $t=29.7$, $\langle Q / N\rangle=84 \%$; (b) intermediate time, $t=1878.0,\langle Q / N\rangle=40 \%$; and (c) late time, $t=88701.1,\langle Q / N\rangle=16 \%$. The particles in this and subsequent figures are shown at $50 \%$ of their actual radius.

Species $A$ is larger and more massive, and consequently has a smaller diffusion coefficient, than species $B$. Thus $Q^{A}(t)$ and $Q^{B}(t)$ have slightly different time dependences, as shown in Fig. 5. The long time motion of the $A$ and $B$ particles is identical; consequently, $Q(t)$-and thus $\chi_{4}(t)$, $g_{4}(r, t)$, and $S_{4}(q, t)$ - do not show a significant difference, 


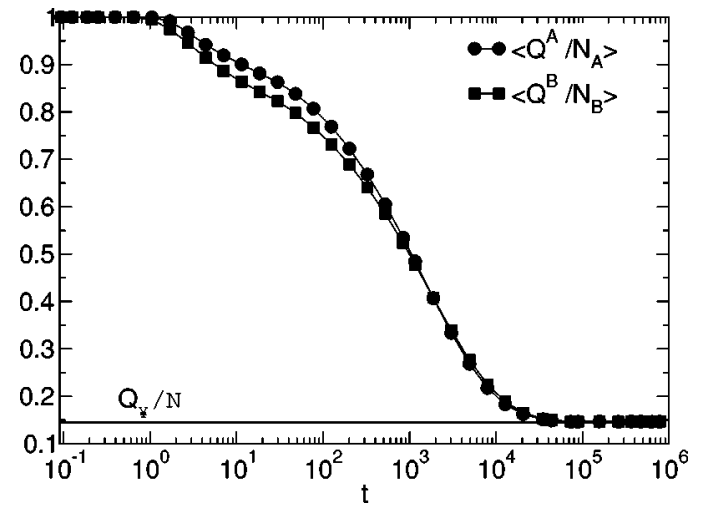

FIG. 5. Time dependence of $Q^{A}(t)$ and $Q^{B}(t)$ for $T=0.60$. The $A$ particles are twice as massive and have an interaction radius 1.2 times larger than $B$; consequently, there is a slight difference in the short-time behavior of $Q(t)$ for the $A$ and $B$ particles.

for the two particle types at times when there are significant correlations in the particles motion.

\section{B. Calculation of $\chi_{4}(t)$}

We show in Fig. 6 the time and temperature dependence of $\chi_{4}(t)$ obtained from Eq. (10) by measuring the sample-tosample fluctuations in $Q(t)$. As found in Refs. 59 and 60, $\chi_{4}(t)$ is zero at short time, is maximum at some intermediate time $t_{4}^{\max }$, and decays at long time to zero in the thermodynamic limit. At $T=2.0$, we see that for all $t \chi_{4}(t)$ is negligible, consistent with our expectation that particle motion is essentially uncorrelated above the temperature at which caging, two-step relaxation, and other supercooled liquid behavior first appears (in this system, at $T \approx 1.0$ ) Thus in subsequent calculations in this paper, we ignore the data corresponding to $T>1.0$.

Insofar as $\chi_{4}(t)$ measures the correlated motion between pairs of particles, calculated equivalently from fluctuations in the number of overlaps or from the four-point correlation function itself, its behavior demonstrates that correlations are time dependent, with a maximum at a time $t_{4}^{\max }$. Similar behavior was reported for the same and other model liquids in Refs. 43, 60, and 77 for a generalized susceptibility related

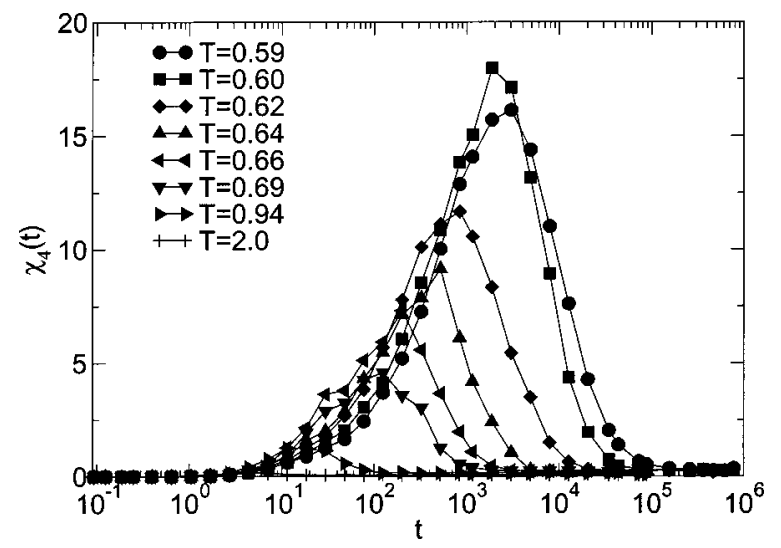

FIG. 6. Time and temperature dependence of $\chi_{4}(t)$. As $T$ decreases, the peak in $\chi_{4}(t)$ monotonically increases and shifts to longer time. We define the time at which $\chi_{4}(t)$ is maximum as $t_{4}^{\max }$.
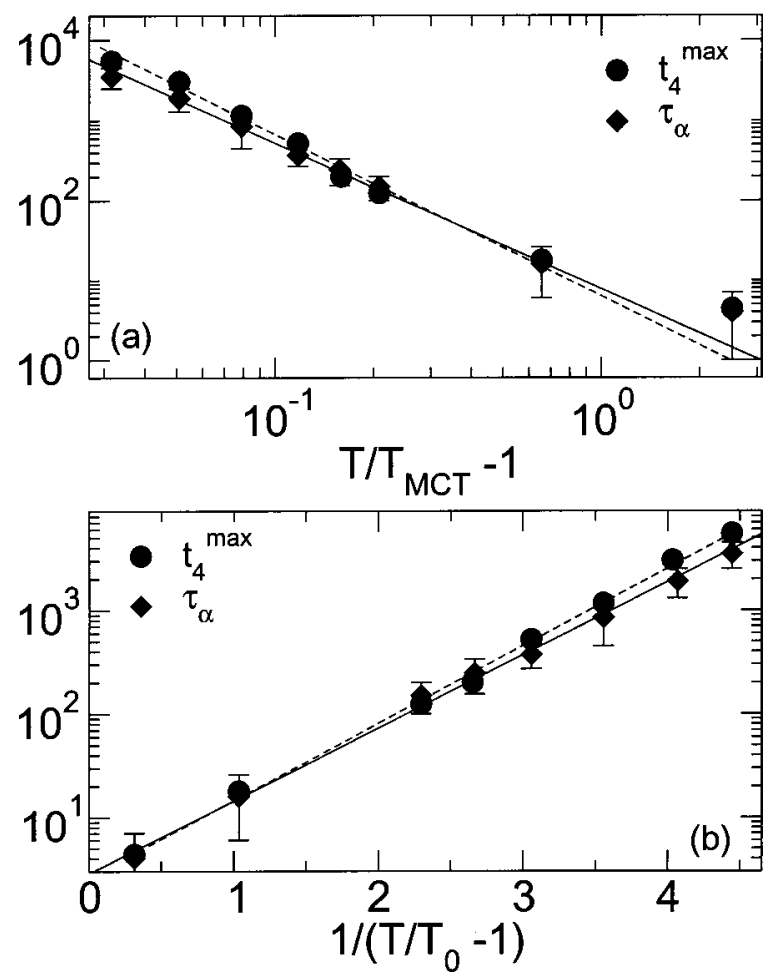

FIG. 7. $T$ dependence of the peak time $t_{4}^{\max }$, compared with $\tau_{\alpha}$. (a) Fits using MCT in the form of Eq. (2). (b) Fits using VFT in the form of Eq. (3). Error bars are shown only for $\tau_{\alpha}$ for clarity. Error bars on $\chi_{4}(t)$ are comparable in size, and are shown in later figures.

to a displacement-displacement correlation function $\chi_{U}(t)$, which measures the correlations between displacements of particles as a function of time. In these works, SHD was observed to be most pronounced in the $\alpha$-relaxation regime. We find that the correlations measured by $\chi_{4}(t)$ are also most pronounced in the $\alpha$-relaxation regime.

We show the $T$ dependence of $t_{4}^{\max }$ together with $\tau_{\alpha}$ in Fig. 7. The two characteristic times have similar $T$ dependence, but $t_{4}^{\max }$ appears to increase slightly more rapidly with decreasing $T$. We note that in Ref. 60 where a much smaller system (500 particles) was studied, $t_{4}^{\max }$ and $\tau_{\alpha}$ was reported to have (roughly) the same $T$ dependence, whereas here we find a small difference between $t_{4}^{\max }$ and $\tau_{\alpha}$ at the lowest temperatures.

Several functional forms are typically used to fit characteristic times of supercooled liquids; some, such at the VFT form of Eq. (3), are motivated on primarily empirical grounds, while others, such as the power law of Eq. (2), are predicted by more complicated theoretical constructions. ${ }^{59}$ We consider both of these forms, and find that we can rea-

TABLE II. Fitting parameters for the power law and exponential form $\tau_{\alpha}$ and $t_{4}^{\max }$

\begin{tabular}{ccc}
\hline \hline Parameters & $\tau_{\alpha}$ & $t_{4}^{\max }$ \\
\hline$\gamma$ & $1.86 \pm 0.03$ & $2.03 \pm 0.01$ \\
$t_{\mathrm{MCT}}$ & $7.58 \pm 0.54$ & $6.31 \pm 0.54$ \\
$A$ & $1.55 \pm 0.05$ & $1.71 \pm 0.07$ \\
$t_{\mathrm{VFT}}$ & $3.59 \pm 0.61$ & $2.66 \pm 0.56$ \\
\hline \hline
\end{tabular}




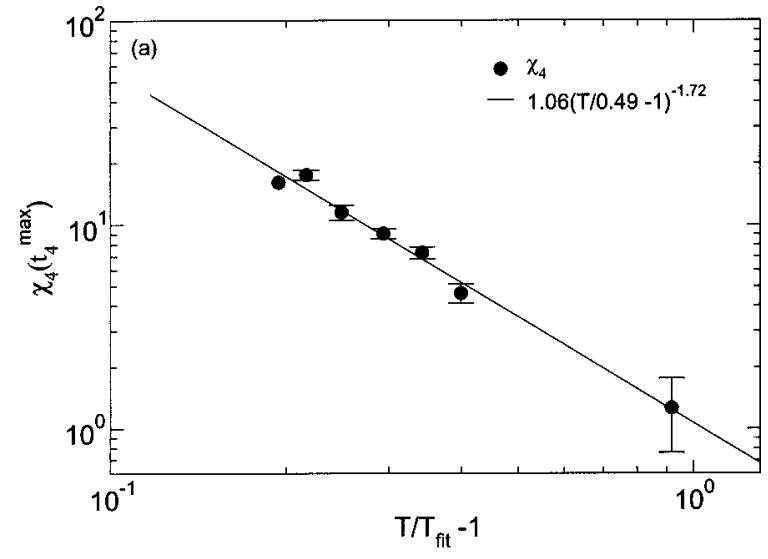

FIG. 8. $T$-dependence of the maximum value of $\chi_{4}(t)$. Power law fit using Eq. (2). We omit the error bar for the lowest temperature $T=0.59$ because of the possible finite size effect, which would contribute additional error.

sonably fit all three characteristic times in the range 0.60 $<T<0.94$ using either a power law or VFT form. Note that all fits deviate from power law as we approach $T_{\mathrm{MCT}}$, where the predictions of MCT are known to break down due to the onset of so-called activated processes not accounted for by the theory. In our fits, we exclude the lowest temperature $T$ $=0.59$.

We performed fitting of the data shown in Fig. 7 using $t(T)=t_{\mathrm{MCT}}\left(T / T_{\mathrm{MCT}}-1\right)^{-\gamma}$ for the power law and $t(T)$ $=t_{\mathrm{VFT}} \exp \left(A /\left(T / T_{0}-1\right)\right)$ for the exponential form. The values for $T_{\mathrm{MCT}}$ and $T_{\mathrm{VFT}}$ are previously determined in Sec. II. We show the fitting parameters in Table II.

The $T$-dependence of the peak value $\chi_{4}\left(t_{4}^{\max }\right)$ is shown in Fig. 8. We observe a monotonic increase in the maximum value of $\chi_{4}$ in the relatively narrow temperature range we study, indicating the increased tendency toward correlated motion on cooling.

Previous studies found that $\chi_{4}\left(t_{4}^{\max }\right)($ Refs. 59, 60) and related susceptibilities ${ }^{43,45}$ may be fit with a power law, $\chi_{4}\left(t_{4}^{\max }\right) \propto\left(T / T_{\mathrm{MCT}}-1\right)^{-\gamma}$. Analytical prediction of Ref. 59 yields $\gamma=1 / 2$. Within numerical accuracy, we may fit $\chi_{4}\left(t_{4}^{\max }\right)$ with the power law, but we do not have a reasonable fit to the power law using $\gamma=1 / 2$ and $T_{\mathrm{MCT}}$ as fitting parameters. We can also fit the data reasonably with a VFT expression, although there is no theory suggesting $\chi_{4}(t)$ should follow this form, and thus within the limited accuracy and temperature range of our simulations, we cannot determine unequivocally the functional form of the $T$ dependence of $\chi_{4}\left(t_{4}^{\max }\right)$.

In Fig. 8 we show the fit of $\chi_{4}\left(t_{4}^{\max }\right)$ using the expression $\chi_{4}\left(t_{4}^{\max }\right)=C\left(T / T_{\mathrm{fit}}-1\right)^{-\gamma_{1}}$ and determined the parameters $C$ $=1.06 \pm 0.02, \quad T_{\text {fit }}=0.49 \pm 0.01$, and $\gamma_{1}=1.73 \pm 0.02$. We performed the fitting for $0.59<T<0.94$. We note that $T_{\text {fit }}$ is within the error bars of the value for $T_{0}$ obtained from $\tau_{\alpha}$. We do not imply that these two temperatures are the same, but it is interesting that we obtain the best fit to a power law of $\chi_{4}\left(t_{4}^{\mathrm{max}}\right)$ for this value of $T_{\text {fit }}$. If we were to choose $T_{\text {fit }}$ $=T_{\mathrm{MCT}}$, and fit $\chi_{4}\left(t_{4}^{\mathrm{max}}\right)$ to a power law, that fit is not acceptable, and we do not show it. The error bars are confidence intervals obtained as a result of fitting $\tau_{\alpha}(T)$ to a power law and exponential form, respectively.

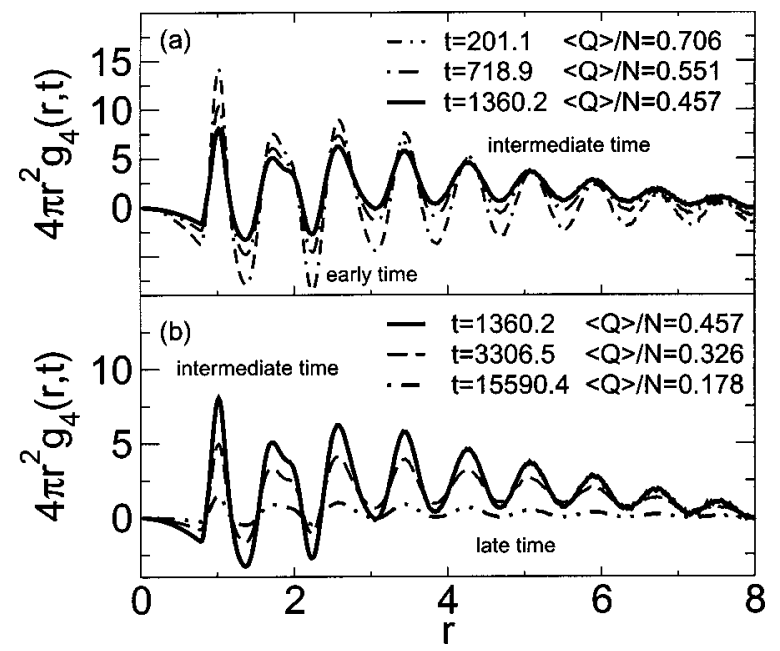

FIG. 9. Time dependence of the four-point, time-dependent density correlation function $g_{4}(r, t)$ at $T=0.60$. (a) Correlations growing in time. (b) Correlations decaying in time. The fractions indicate the average fraction of overlapping particles present at time $t$. We multiply $g_{4}(r, t)$ with $4 \pi r^{2}$ to better reveal its long decaying tail.

\section{Calculation of $g_{4}(r, t)$ and $S_{4}^{o l}(q, t)$}

The radial correlation function $g_{4}(r, t)$ calculated from Eq. (16) is plotted versus $r$ for several $t$ in Fig. 9, at our second coldest temperature $T=0.60$.

The positions of the peaks in $g_{4}(r, t)$ are identical to the positions of the peaks in $g(r)$ (not shown). We confirm that $g_{4}(r, t)=g(r)-1$ in the ballistic regime, where $\langle Q(t) / N\rangle$ $=1$. In the long time limit, $g_{4}(r, t)=\left(Q_{\infty} / N\right)^{2}(g(r)-1)$. Note that in the diffusive regime $g_{4}^{o l}(r, t)$ is the pair correlation function of the random overlaps normalized by $\langle Q(t) / N\rangle^{2}$ to yield $g(r) . g_{4}(r, t)$ deviates from $g(r)-1$ when $\langle Q(t) / N\rangle$ deviates from unity and $\chi_{4}(t)$ becomes nonzero. ${ }^{78}$ The range of $g_{4}(r, t)$ increases with increasing $t$ until a time $t_{4}^{\max }$. At $t_{4}^{\max }, g_{4}\left(r, t_{4}^{\max }\right)$ [indicated by the solid curve in Figs. 9(a) and 9(b)] exhibits a long tail which decreases slowly to zero with increasing distance. For $t$ greater

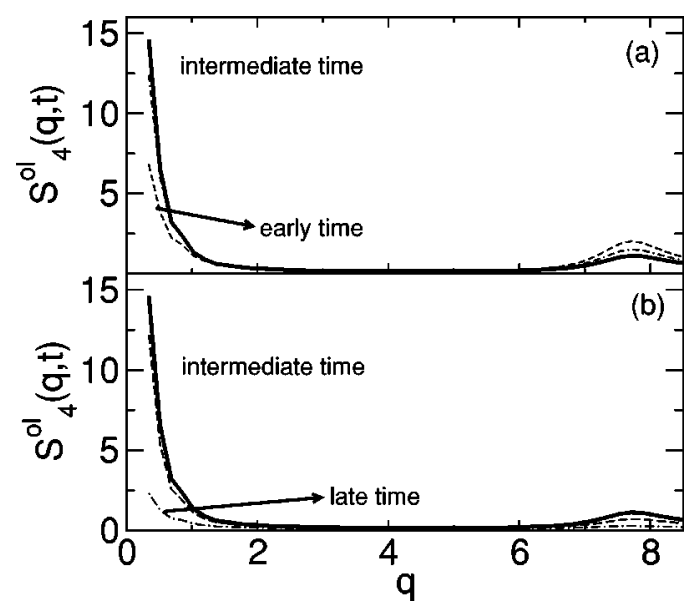

FIG. 10. Time dependence of $S_{4}^{o l}(q, t)$ at $T=0.60 . S_{4}^{o l}(q, t)$ is shown at times identical to those shown for $g_{4}(r, t)$ in Fig. 9. Note that the height of the first diffraction peak in $S_{4}^{o l}(q, t)$ decreases monotonically as a function of time. This is because it depends on the number of overlapping particles, which decreases monotonically in time (see Fig. 3). 


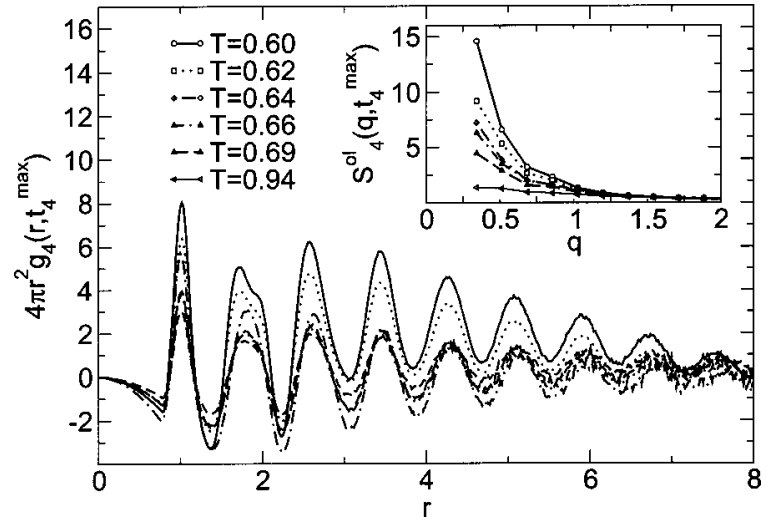

FIG. 11. Temperature dependence of $g_{4}\left(r, t_{4}^{\max }\right)$ at six values of $T$, indicated in the legend. Inset shows the structure factor $S_{4}^{o l}\left(q, t_{4}^{\max }\right)$ for the same values of $T$.

than $t_{4}^{\max }$, the amplitude and range of $g_{4}(r, t)$ decrease, and $g_{4}(r, t)$ becomes zero when $\chi_{4}(t)$ decays to zero (not shown). The positions of the peaks in $g_{4}(r, t)$ do not appear to change with decreasing $T$. \{As a check of our calculation, we numerically integrate $g_{4}^{o l}(r, t) \quad$ [recall $\int d \mathbf{r} g_{4}^{o l}(r, t)$ $\equiv(V / N)^{2}\left\langle Q(t)^{2}\right\rangle$ from Eqs. (10), (13), and (16)] to confirm that the value of $\chi_{4}(t)$ thus obtained is identical to that obtained from Eq. (10) for all $T$.\}

The four-point structure factor of overlapping particles $S_{4}^{o l}(q, t)$ calculated from Eq. (21) is plotted versus $q$ in Fig. 10 , at $T=0.60$. We find that at very early times [when $\langle Q(t) / N\rangle=1] S_{4}^{o l}(q, t)=S(q)$. We find that while $S(q)$ shows no change at small $q$ [see Fig. 1(b)], $S_{4}^{o l}(q, t)$ develops a peak at small $q$ which grows [Fig. 10(a)] and decays in time [Fig. 10(b)], indicating the presence of long-range correlations in the locations of overlapping particles.

Figure 11 shows the $T$ dependence of $g_{4}(r, t)$ at the peak characteristic time $t_{4}^{\max }$ when the correlations at each $T$ are most pronounced, as measured by $\chi_{4}(t)$. The inset of Fig. 11 shows the four-point structure factor $S_{4}^{o l}\left(q, t_{4}^{\max }\right)$, calculated using Eq. (21). We find that $S_{4}^{o l}\left(q, t_{4}^{\max }\right)$ develops a peak at small $q$ that grows with decreasing $T$. This behavior is reminiscent of that observed in $S(q)$ for liquids near a critical point, at which two-point density fluctuations becomes longranged. Here, however, two-point density fluctuations remain small and short ranged. ${ }^{78}$

\section{Calculation of $\xi_{4}(t)$}

Inspired by the Ornstein-Zernike theory OZT, ${ }^{79}$ which describes, e.g., density fluctuations near a liquid-gas transition, we use the following function:

$$
S_{4}^{o l}(q, t)=\frac{S_{4}^{o l}(0)}{\left(1+\left(q \xi_{4}(t)\right)^{2}\right)},
$$

where $S_{4}^{o l}(0)$ and $\xi_{4}(t)$ are fitting parameters. The fitting was performed using an interior-reflective Newton method ${ }^{80}$ in Matlab, and setting the termination tolerance of the function value to 0.1 . Note that this correlation length $\xi_{4}(t)$ ), and all other correlation lengths to be calculated in the reminder of this paper, are calculated from $S_{4}^{o l}(q, t)$ or some appropriate portion thereof. We drop the subscript "ol" for simplicity

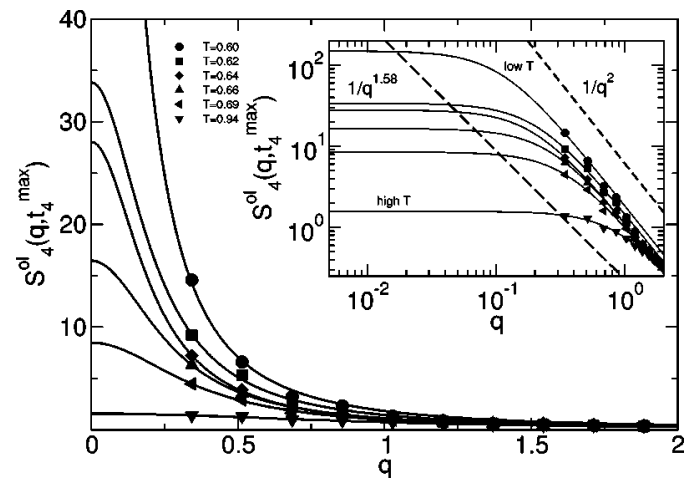

FIG. 12. Small $q$ behavior of $S_{4}^{o l}\left(q, t_{4}^{\max }\right)$. Inset shows a $\log -\log$ plot of $S_{4}^{o l}\left(q, t_{4}^{\max }\right)$ vs $q$. The lines in both figures are fits to the data using Eq. (30). The second lowest temperature $T=0.60$ is difficult to fit because of the large uncertainty in $S_{4}^{o l}(0, t)$ at times in the vicinity of $t_{4}^{\max }$ (see text for further discussion).

in notation. We find a good fit to the data in the $q$ range from $q=0.34$ to $q=1.9$, shown by the lines in Fig. 12, for each $T$ and time. This range of data corresponds to the range 3.3 $<r<L$, in contrast to the range $1.7<r<7$ used in Ref. 61 . The observed narrowing of the peak directly reveals the growing range of $g_{4}(r, t)$ with decreasing $T$.

The inset of Fig. 12 shows $S_{4}^{o l}\left(q, t_{4}^{\max }\right)$ plotted logarithmically versus $q$. The two dashed lines are the prediction of Ref. 54 for fragile $\left(1 / q^{1.58}\right)$ and strong $\left(1 / q^{2}\right)$ glassformer. We see that approaching $T_{\mathrm{MCT}}$, our data more closely aligns with the Garrahan-Chandler prediction for a strong glassformer, even though the LJ mixture we study was shown previously $^{62}$ to be a fragile liquid of intermediate fragility (less fragile than the 80:20 Kob-Anderson system ${ }^{82}$ ).

The time and temperature dependence of $\xi_{4}(t)$ obtained from this fit is plotted for several state points in Fig. 13. We see that the qualitative behavior of $\xi_{4}(t)$ is similar to that of $\chi_{4}(t): \xi_{4}(t)$ has a maximum in time that coincides with the maximum in $\chi_{4}(t)$, and as $T$ decreases, the amplitude and time of this maximum increase. The highest values of $\xi_{4}(t)$ for $T=0.60$ exceed half the simulation box size. The fit at these points depends strongly on the number of points used, initial parameter guesses, and other details and can yield

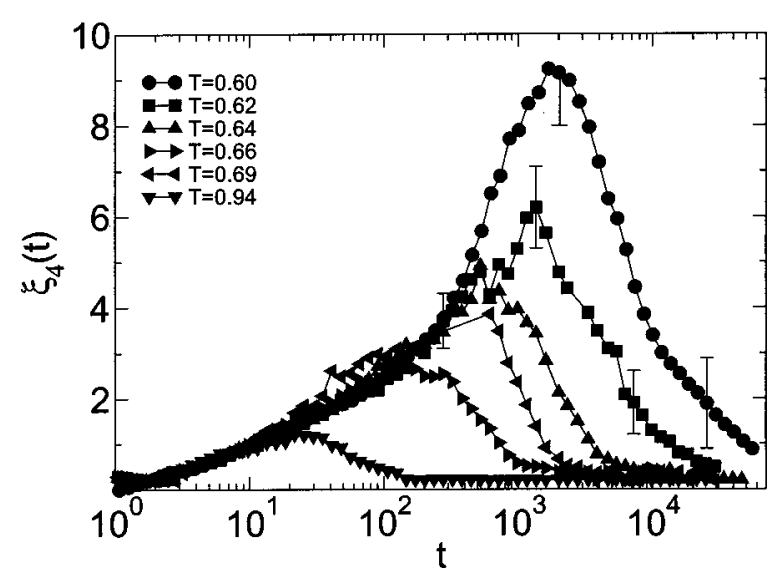

FIG. 13. Time and temperature dependence of $\xi_{4}(t)$ obtained from the fits to Eq. (30). The data shown are smoothed over successive groups of three points. 

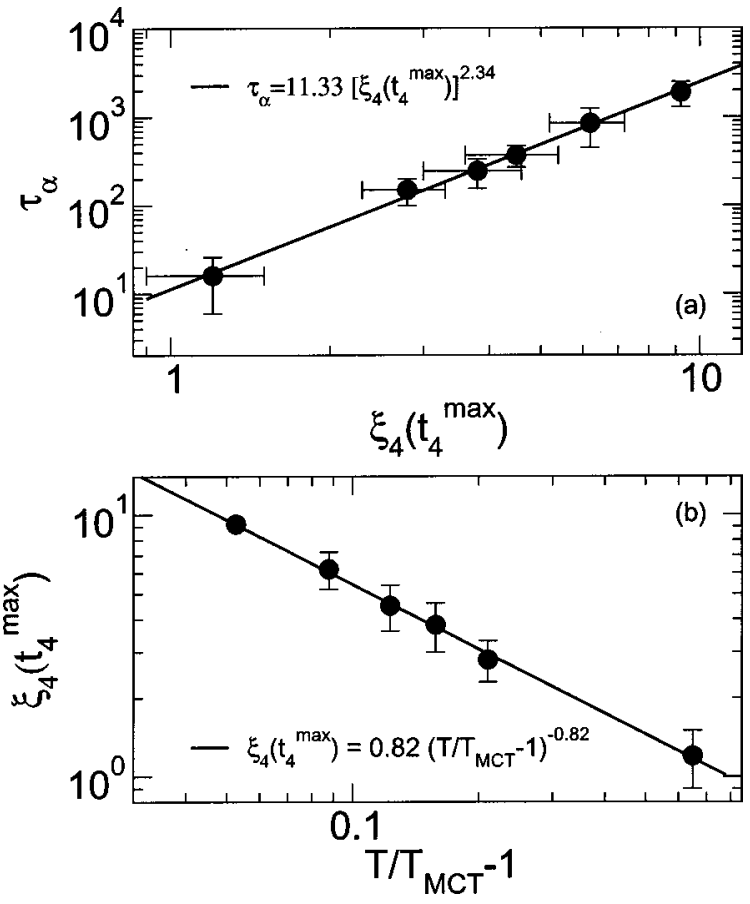

FIG. 14. (a) Log-log plot of $\tau_{\alpha}$ vs $\xi_{4}\left(t_{4}^{\max }\right)$. (b) Log-log plot of $\xi_{4}\left(t_{4}^{\max }\right)$ vs $T / T_{\mathrm{MCT}}-1$. Solid lines are power law fits using equations similar to Eq. (2).

large values (e.g., $>40$ ) depending on these details. Since these values greatly exceed the range over which we can meaningfully interpret the resulting correlation length, we make no attempt to rigorously define the upper error bounds at these points, but the data is well bounded from below. The fits at all other points and temperatures are well constrained. The length scale $\xi_{4}(t)$ characterizes the typical distance over which "overlapping" particles are spatially correlated.

In Ref. 61, we calculated a correlation length $\xi_{4}^{\text {env }}(t)$ from $g_{4}^{*}(r, t)$ by fitting directly the maxima of $g_{4}^{*}(r, t)$ in the range $1.7<r<7$ to an exponential envelope function $f(r)$ $=a \exp \left[-r / \xi_{4}\right.$ env $\left.(t)\right]$ (the "envelope" method). Because of the narrowness of that range relative to the range considered in the present work, $\xi_{4}^{\text {env }}(t)$ greatly underestimates the extent of the correlation between overlapping particles measured by $\xi_{4}(t)$. We see that the qualitative behavior of $\xi_{4}(t)$ is similar to $\xi_{4}(t)$ obtained from the envelope fit in Ref. 61, but the magnitude of the lengths obtained with the present method are substantially larger due to the fact that the present correlation length captures the long-ranged correlations of overlapping particles.

\section{E. Relationship between $\xi_{4}\left(t_{4}^{\max }\right)$ and $\tau_{\alpha}$}

In a study of dynamical heterogeneity in the Ising spin glass, ${ }^{84}$ the authors found that the spatial correlation of single-site relaxation times obtained from local spin autocorrelation functions, provide a length scale for dynamical heterogeneity, and that length scale grows with decreasing temperature. The large statistical error in $\xi_{4}\left(t^{\max }\right)$, combined with the short range of $T-T_{\mathrm{MCT}}$ over which the simulations are performed make determination of the functional form describing the dependence of $\xi_{4}\left(t^{\mathrm{max}}\right)$ on $T-T_{\mathrm{MCT}}$ problematic. Instead, we examine the dependence of the relaxation

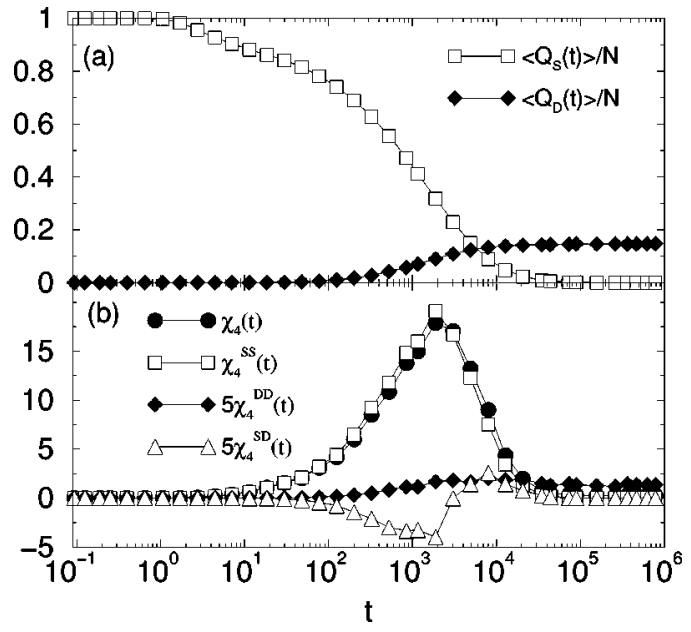

FIG. 15. (a) Self and distinct contributions to $Q(t)$ and (b) self, distinct, and self-distinct contributions to $\chi_{4}(t)$ at $T=0.60$. We see that $\chi_{4}^{S D}(t)$ is negative for most times, demonstrating the tendency for localized and replaced particles to be anticorrelated. In this figure the values of $\chi_{4}^{S D}(t)$ and $\chi_{4}^{D D}(t)$ are enlarged by a factor of 5 for the purpose of clarity.

time $\tau_{\alpha}$ versus $\xi_{4}\left(t^{\max }\right)$, since $\tau_{\alpha}$ spans nearly three orders of magnitude in time. Figure 14(a) demonstrates that $\tau_{\alpha}$ and $\xi_{4}\left(t^{\mathrm{max}}\right)$ are related to each other by a power law: $\tau_{\alpha}$ $\sim \xi_{4}\left(t^{\max }\right)^{2.34}$. This result agrees with result results found for the facilitated Ising model, in which the correlation length and relaxation time were found to be related by a power law over six decades in time. ${ }^{83}$ In Ref. 48 , the authors compared the "bond breakage" relaxation time (which is likely proportional to the relaxation time of the self-intermediate scattering function) to the correlation length, and they found a power law with exponent 2 , close to the value of (2.34) obtained here.

In the model liquid under study, $\tau_{\alpha}$ is known to to follow a power law, $\tau_{\alpha} \sim\left(T / T_{\mathrm{MCT}}-1\right)^{-1.86}$ (see Table II), for $T$ approaching $T_{\mathrm{MCT}}$ from above. Combining $\tau_{\alpha} \sim \xi_{4}\left(t^{\max }\right)^{2.34}$ and $\tau_{\alpha} \sim\left(T / T_{\mathrm{MCT}}-1\right)^{-1.86}$, we obtain $\xi_{4}\left(t^{\max }\right) \sim\left(T / T_{\mathrm{MCT}}\right.$ $-1)^{-0.79}$. As shown in Fig. 14(b), a power law fit of $\xi_{4}\left(t^{\max }\right)$ versus $T / T_{\mathrm{MCT}}-1$ gives an exponent $0.82 \pm 0.02$, which is within numerical error of the value predicted.

\section{F. Self and distinct contributions to $Q(t), \chi_{4}(t)$, $g_{4}(r, t)$, and $S_{4}(q, t)$}

To obtain a clearer physical picture of dynamical heterogeneity in our model supercooled liquid, we next examine the self and distinct contributions to $Q(t), \chi_{4}(t), g_{4}(r, t)$, and $S_{4}(q, t)$. Recall that particles that are within a distance $a$ of their original position at time $t$-localized particlescontribute to the self part of these functions, while particles that move and are replaced by another particle contribute to the distinct part. We show the self and distinct parts of $Q(t)$ for $T=0.60$ in Fig. 15(a). For $t \leqslant 100, Q_{D}(t)=0$ and thus $Q(t)=Q_{S}(t)$; in other words, over this time range, some particles have moved beyond a distance $a$ of their original position, but no particle has yet been replaced by another particle. $Q_{S}(t)$ decays to zero at late times, because all particles eventually become "delocalized" (i.e., move a distance greater than $a$ ). It is interesting to note that for each $T$, the 


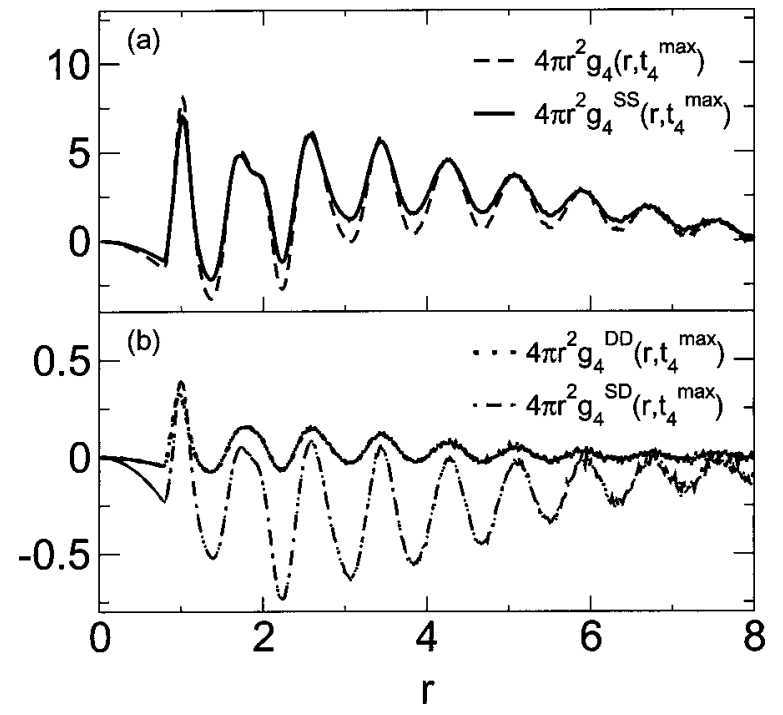

FIG. 16. (a) $g_{4}^{S S}\left(r, t_{4}^{\max }\right)$ (solid line) at $T=0.62$. The dashed line shows $g_{4}(r, t)$ for comparison. (b) Distinct (dotted line), and self-distinct (dotdashed line) parts of $g_{4}\left(r, t_{4}^{\mathrm{max}}\right)$ at $T=0.62$. We separate the contributions into two panels due to the large difference in amplitude.

fraction of particles that have not yet moved at a time equal to the relaxation time $\tau_{\alpha}$ is roughly $50 \%$. At long times, $Q_{D}(t) / N=\rho V_{a}$, corresponding to the fraction of random overlaps in the system, as explained in Sec. III.

Reference 60 showed that $\chi_{4}(t)$ is dominated at all times by $\chi_{4}^{S S}(t)$, and we observe this in Fig. 15(b). In fact, the value of $\chi_{4}^{S S}\left(t_{4}^{\mathrm{max}}\right)$ is slightly greater than $\chi_{4}\left(t_{4}^{\max }\right)$. Thus the growing fluctuations in the number of overlapping particles is dominated by increasing fluctuations in the number of localized particles. In contrast, $\chi_{4}^{D D}(t)$, which measures the fluctuations in the number of particles that are replaced in a time $t$, is small at all times. These "replaced" particles may include particles that "follow" other particles in a stringlike fashion. ${ }^{46}$ As was shown in Ref. 46, the average length of these strings grows slowly with decreasing $T .{ }^{47}$ Finally, $\chi_{4}^{S D}(t)$, which measures the cross-correlation between localized and replaced particles, is negative at intermediate times, demonstrating the tendency for localized and replaced particles to be anticorrelated (i.e., spatially separated), as shown for the most mobile and immobile particles at intermediate times in a similar model liquid in Ref. 44.

In Fig. 16 we show $g_{4}^{S S}\left(r, t_{4}^{\max }\right), g_{4}^{D D}\left(r, t_{4}^{\max }\right)$, and $g_{4}^{S D}\left(r, t_{4}^{\max }\right)$ for $T=0.60$. As expected from the fact that $\chi_{4}(t) \approx \chi_{4}^{S S}(t)$, the largest contribution to $g_{4}(r, t)$ is from localized particles as measured by $g_{4}^{S S}(r, t)$. In contrast, a much weaker correlation [compared to $g(r)-1$ ] is exhibited by $g_{4}^{D D}(r, t)$ [Fig. 16(b)]; note difference in values on $y$-axis from those in Fig. 16(a). The anticorrelation between localized and replaced particles is evident in the negative values attained by $g_{4}^{S D}(r, t)$ for nearly all $r$.

We next calculate the correlation length associated with each contribution to $g_{4}(r, t)$ using the OZT method. Since $g_{4}(r, t), \chi_{4}(t)$, and $S_{4}(q, t)$ are dominated by their self parts, it is not surprising that we find $\xi_{4}^{S S}(t)$ (not shown) to be very similar to $\xi_{4}(t)$, and with a slightly higher amplitude. $\xi_{4}^{S S}(t)$ itself is also a fundamentally important length since it quan-
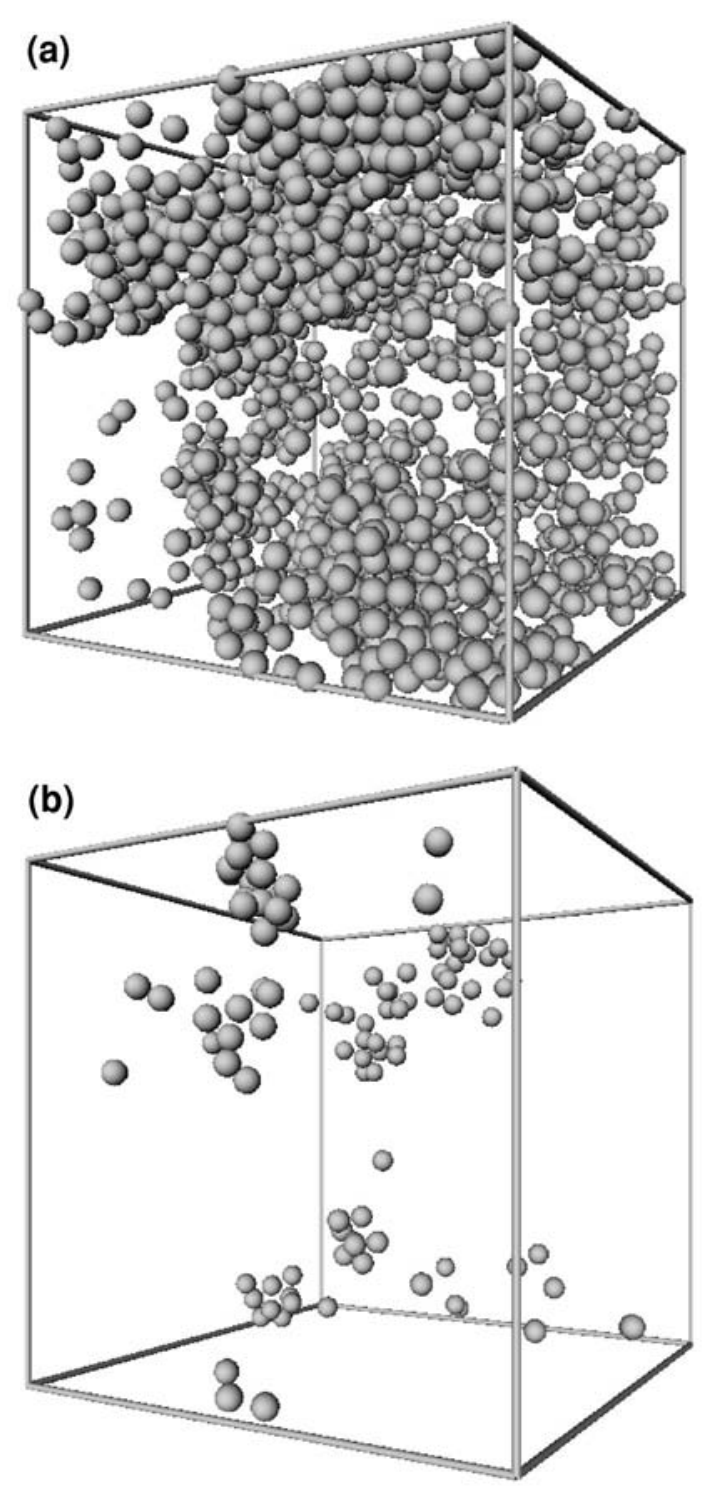

FIG. 17. (a) Snapshot of localized particles at $t=t_{4}^{\max }$ and $T=0.60$. The fraction is $\left\langle Q_{S} / N\right\rangle=0.244$. (b) Snapshot of localized particles at $t$ $=0.03 t_{4}^{\max }$ and $T=0.60 .\left\langle Q_{S} / N\right\rangle=0.01$.

tifies correlations of localized domains in glassforming liquids. We show a snapshot of localized particles at $T=0.60$ and $t_{4}^{\max }$ in Fig. 17.

In Fig. 18, we show the correlation length $\xi_{4}^{D D}(t)$ corresponding to replaced particles. We show $\xi_{4}^{D D}(t)$ only for those values of $t$ where it can be reasonably estimated. At early times only a small fraction of particles are replaced by other particles, as can be seen in the inset of Fig. 18. This results in a noisy signal in $S_{4}^{D D}(r, t)$ at early times, and estimation of the correlation length becomes more difficult at those times. We do observe that $\xi_{4}^{D D}(t)$ slightly increases at intermediate times that are shorter then $t_{4}^{\max }$.

We show a snapshot of replaced particles at $T=0.60$ and $t_{4}^{\max }$ in Fig. 19. We expect that some fraction of these particles exist in groups ("strings"), as was shown in Ref. 46, in which successive particles replace a neighboring particle. In a related study of a different glassforming liquid, ${ }^{85}$ stringlike motion was found to be most prominent in the late- $\beta /$ early- $\alpha$ 


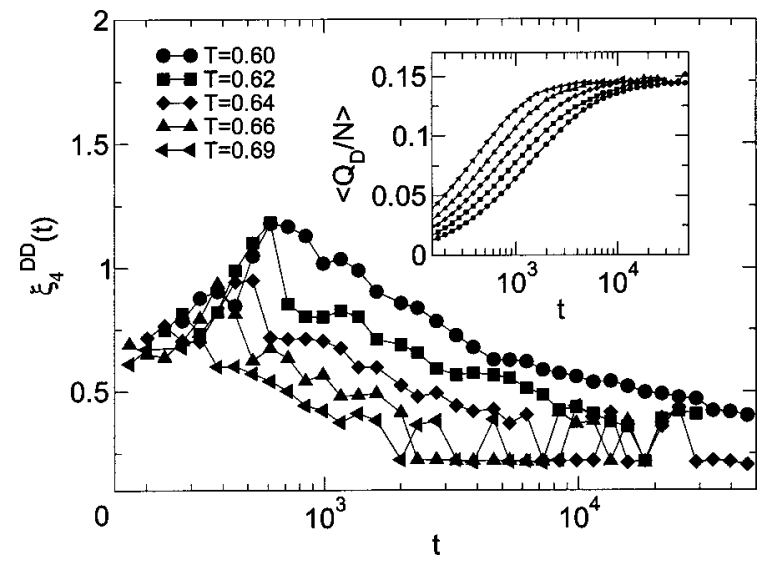

FIG. 18. Time and temperature dependence of $\xi_{4}^{D D}$. We show $\xi_{4}^{D D}(t)$ only for those $t$ where it can be reasonably estimated. Inset shows $\left\langle Q_{D}(t) / N\right\rangle$ at the times when we can reasonably estimate $\xi_{4}^{D D}(t)$.

relaxation regime, consistent with the present findings. The connection between replaced particles and strings will be discussed elsewhere.

Finally, we do not show $\xi_{4}^{S D}(t)$ because the signal from $S_{4}^{S D}(q, t)$ is inadequate due to small signal to noise ratio for small $q$ values of $S_{4}^{S D}(q, t)$.

\section{G. Spatial correlations of localized and delocalized particles}

We next consider the four-point correlation functions corresponding to localized particles [described by the self term in $Q(t)$ ] and to delocalized particles, defined as the subset of non-self-overlapping particles. As we discussed in Sec. III, $\chi_{4}^{D L}$ of delocalized particles is mathematically identical to the susceptibility $\chi_{4}^{S S}$ for localized particles, which we verified. Since these susceptibilities are the volume integrals of $g_{4}(r, t)$, one might expect $g_{4}^{D L}(r, t)$ and $g_{4}^{S S}(r, t)$, as

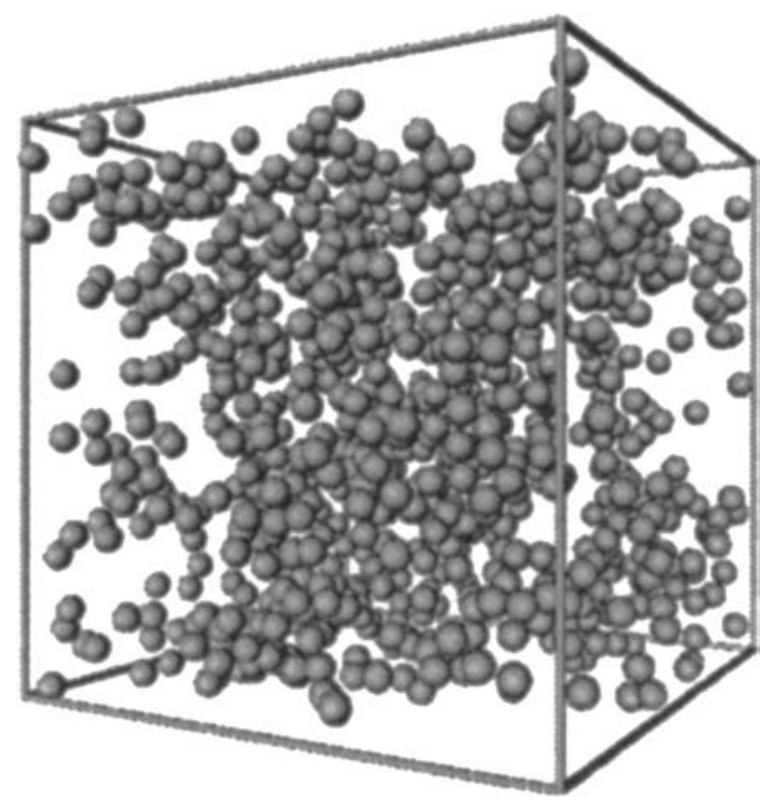

FIG. 19. Snapshot of replaced particles at $t_{4}^{\max }$. Some fraction of these particles are participating in "strings" for $T=0.60,\left\langle Q_{D} / N\right\rangle=0.114$.

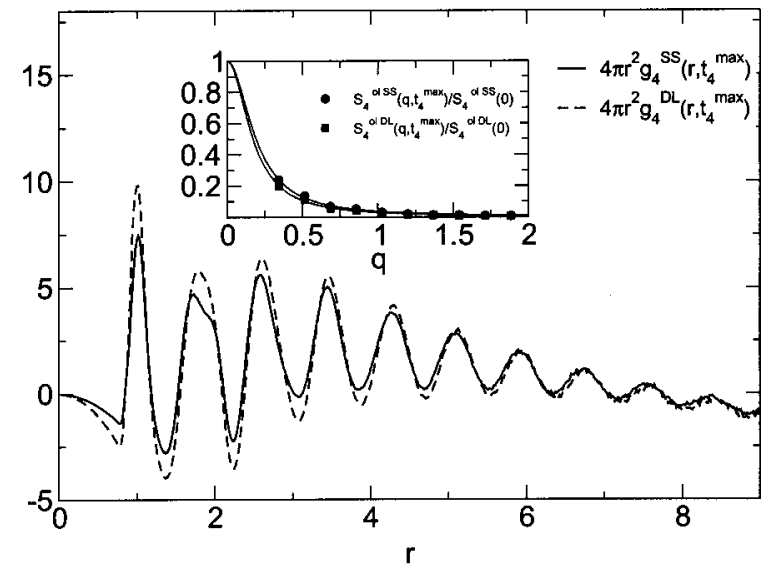

FIG. 20. (a) Four-point correlation functions corresponding to localized particles, $g_{4}^{S S}(r, t)$, and delocalized particles, $g_{4}^{D L}(r, t)$, at the peak time for $T=0.62$. At this $t$, there are, on average, $41 \%$ localized and $59 \%$ delocalized particles. We multiply $g_{4}^{S S}(r, t)$ and $g_{4}^{D L}(r, t)$ with $4 \pi r^{2}$ to better reveal their tails. Inset shows $S_{4}^{\text {ol } S S}\left(q, t_{4}^{\max }\right)$ and $S_{4}^{o l} D L\left(q, t_{4}^{\max }\right)$. There is no significant difference between corresponding correlation lengths.

well as $\xi_{4}^{D L}(t)$ and $\xi_{4}^{S S}(t)$, are also identical. Mathematically, however, they need not be identical. Instead, one would also intuitively expect that delocalized particles, since they move, should be spatially distinct from localized particles. To explore these possibilities, we first compare $g_{4}^{D L}(r, t)$ and $g_{4}^{S S}(r, t)$ at the peak time in Fig. 20. The functions are different up to the fourth nearest neighbor distance, and show a marked difference in the first and second neighbor peaks. This means that the short-range spatial correlations of localized particles is different from that of delocalized particles. The split second peak is absent in $g_{4}^{D L}(r, t)$, but pronounced in $g_{4}^{S S}(r, t)$. Since a split second peak is often associated with close-packed amorphous structure, this supports the notion that localized particles are "better packed" than delocalized particles as observed in Refs. 16 and 44. The long-range structure of localized and delocalized particles appears to be the same, i.e., a nearly isotropic liquid.

We next consider the correlation lengths $\xi_{4}^{S S}(t)$ and $\xi_{4}^{D L}(t)$, calculated using the OZT method of fitting to $S_{4}^{\text {ol } S S}(q, t)$ and $S_{4}^{\text {ol } D L}(q, t)$ at small $q$ values, respectively.

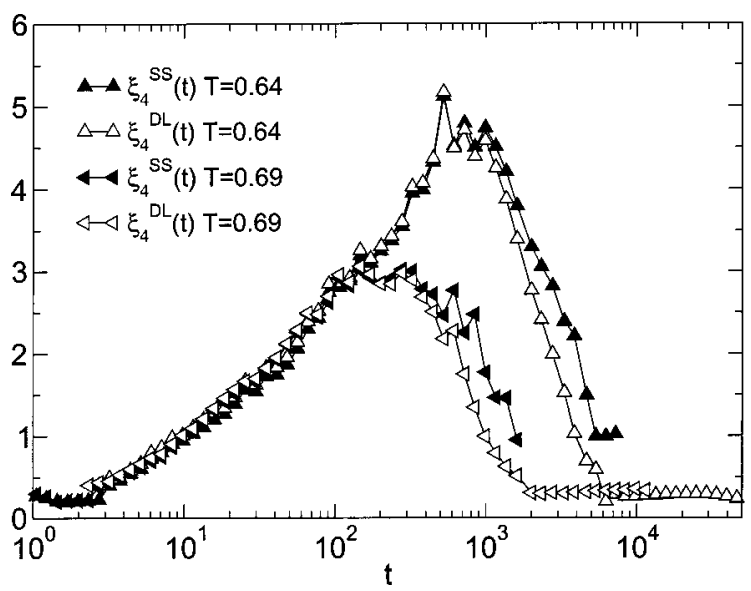

FIG. 21. Time dependence of dynamical correlation lengths $\xi_{S S}$ and $\xi_{D L}$ at $T=0.64$ and $T=0.69$. 


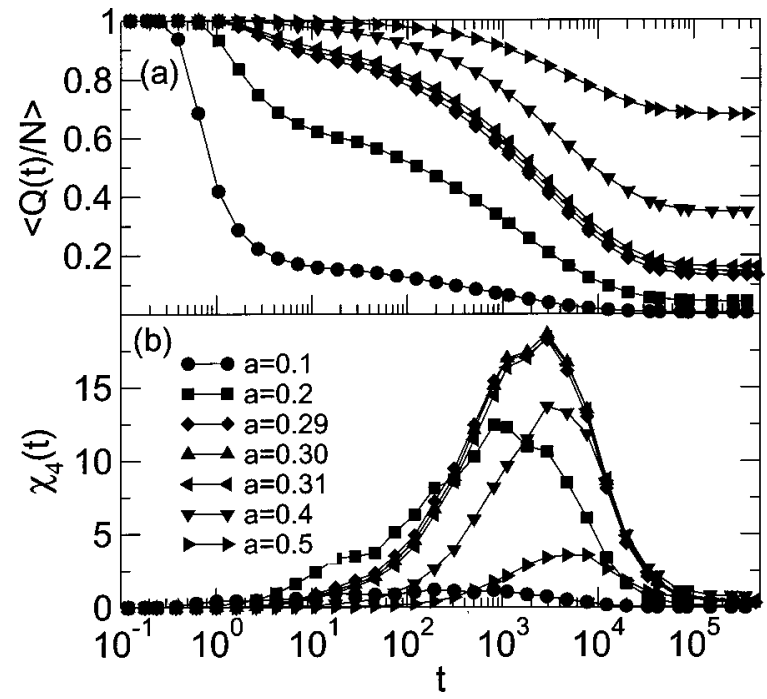

FIG. 22. (a) $Q(t)$ as a function of $a$ at $T=0.59$ for $a=0.1, a=0.2,0.29$, $0.3,0.31,0.4$, and 0.5. (b) $\chi_{4}(t)$ as a function of $a$. The position of the peak in $\chi_{4}(t)$ for $a=0.29, a=0.30$, and $a=0.31$ is the same. The amplitude in $\chi_{4}(t)$ is strongest for $a=0.3$.

We find that $\xi_{4}^{D L}(t)$ and $\xi_{4}^{S S}(t)$ have the same time and $T$-dependence, as can be seen in Fig. 21, which is not surprising since $\chi_{4}^{D L}(t)$ and $\chi_{4}^{S S}(t)$ must be identical and both $\chi_{4}(t)$ and $\xi_{4}(t)$ are similarly defined in terms of an integral of $g_{4}(r, t)$.

\section{DISCUSSION}

In this paper, we calculated a four-point, time-dependent density correlation function $g_{4}(r, t)$ and corresponding time dependent structure factor $S_{4}(q, t)$, and demonstrated that those functions are sensitive to correlated motion and dynamical heterogeneity in a model glassforming liquid. As derived in previous works, ${ }^{58-61,70}$ this correlation function is related to an order parameter $Q(t)$ corresponding to the number of "overlapping" particles in a time window $t$, where the term "overlap" is used to denote a particle which was either localized or replaced in a time $t$.

We calculated the correlation length $\xi_{4}(t)$, characterizing the range of $g_{4}^{o l}(r, t)$, and showed that it depends on time, and attains its maximum value in the $\alpha$-relaxation regime. We also showed that this maximum grows to nine particles diameters, surpass a half of the simulation box size, close to $T_{\mathrm{MCT}}$. This length scale characterizes the typical size of dynamically homogeneous domains. While we cannot reliably predict the behavior of $\xi_{4}\left(t_{4}^{\max }\right)$ at lower $T$, we find no tendency for slowing down of its growth. These findings are consistent with calculations of characteristic length scales from the displacement-displacement correlation function, ${ }^{76}$ cluster size ${ }^{85}$ and other measures of correlated particle motion and dynamical heterogeneity. ${ }^{48,50,51}$

We showed that $\xi_{4}(t)$ is dominated by localized particles, but is essentially the same as that obtained by considering the delocalized particles (the set of particles that in any time window $t$ move beyond a distance $a$ ) due to the mathematical identity between $\chi_{4}$ for localized and delocalized particles. This suggests a picture of fluctuating domains of temporarily localized and delocalized particles, perhaps similar to that proposed by Stillinger and Hodgedon. ${ }^{9}$ We know from previous simulation studies (see Ref. 44) that the most mobile particles in any time window (a subset of the set of delocalized particles) exhibit quasi-one-dimensional motion and form highly ramified clusters, and thus the highly mobile regions of our fluid are not compact. A previous study ${ }^{16}$ of the most immobile particles on the time scale of the late- $\beta /$ early- $\alpha$ relaxation (a subset of the set of localized particles) showed these particles to be much more compact than their mobile counterparts, consistent with the relatively compact structure of localized particles seen in the present study.

Recently, van Zon and Schoefield ${ }^{86}$ derived multipoint and multitime correlation functions within mode coupling theory, and compared their results with those obtained with standard mode coupling theories in which an assumption of Gaussian density fluctuations is made. ${ }^{1}$ They demonstrated that non-Gaussian distribution of force fluctuation gives an important contributions to higher-order correlation functions. In a subsequent paper, ${ }^{87}$ they applied their mode coupling theory of higher order correlation functions to a hard sphere system. They showed that their results are in excellent agreement with the simulation, demonstrating the importance of considering higher-order correlation functions in examining the microscopic origins of complex relaxation behavior and dynamical heterogeneities.

Finally, we note that all quantities presented here can be measured in dense colloidal suspensions using confocal microscopy studies. ${ }^{32,33}$

\section{ACKNOWLEDGMENTS}

We thank the National Partnership for Advanced Computing Infrastructure (NPACI) program and the University of Michigan Center for Advanced Computing for generous amounts of CPU time on the University of Michigan AMD Athlon cluster. We thank J. P. Garrahan and D. Chandler for interesting discussions that led to a comparison of our data with their theoretical prediction in Fig. 12. We thank J. W. Palko, Y. Gebremichael, and M. Vogel for useful discussions. This work was performed in partial fulfillment of N.L.'s dissertation requirements.

\section{APPENDIX: THE CHOICE OF PARAMETER a}

It is natural to ask how the quantities calculated in this paper are affected by the choice of the parameter $a$, and what is an optimal choice of $a$ ?

In this paper, we are interested in quantifying correlated particle motion. If we choose $a$ too small, then the shorttime, vibrational motion of the particles, which is known to be only weakly correlated, will be included in our analysis. One estimate of the amplitude of vibrational motion is the plateau value of the mean square displacement $\left\langle r^{2}\right\rangle_{\text {plateau }}$. Hence we wish to chose $a$ larger than $\left\langle r^{2}\right\rangle_{\text {plateau }}^{1 / 2}$. For the state points simulated, we find $\left\langle r^{2}\right\rangle_{\text {plateau }}^{1 / 2} \simeq 0.25$ with a relatively weak $T$-dependence [Fig. 2(a)]. On the other hand, if we choose a large value of $a$, then one particle can unphysically "overlap" with two or more particles at a later time. In 
this case we count too many overlaps, and $Q(t)$ becomes greater than the number of particles in the system. This is the case, e.g., for $a=0.8$.

Figure 22 shows $Q(t)$ and $\chi_{4}(t)$ at the second lowest temperature $T=0.60$ for several values of $a$. Since $Q_{p}(t)$ $=\lim _{a \rightarrow 0} Q(t)$, the result for $a=0.1$ is similar to what we expect for point particles, namely, $Q(t) / N$ quickly relaxes to $N V_{a} / V$, where $V_{a}=4 / 3 \pi a^{3}$, on a time scale much smaller than that of the $\alpha$-relaxation time, ${ }^{63}$ and $\chi_{4}(t)$ is small. For intermediate values of $a$, we see that $\chi_{4}(t)$ gives the greatest amplitude for $a=0.3$, as originally suggested in Ref. 59 . Thus we use $a=0.3$ for our analysis throughout this paper. We do not expect choosing a slightly larger or slightly smaller would qualitatively alter any of our conclusions.

${ }^{1}$ W. Gotze, in Liquids, Freezing, and the Glass Transition, edited by D. Levesque, J. P. Hansen, and J. Zinn-Justin (Elsevier, New York, 1991).

${ }^{2}$ P. G. Debenenedetti, Metastable Liquids: Concepts and Principles (Princeton University Press, Princeton, NJ, 1996), Chap. 4.3, and references therein.

${ }^{3}$ T. R. Kirkpartick and P. G. Wolynes, Phys. Rev. A 35, 3072 (1987); Phys. Rev. B 36, 8552 (1987); K. Kree, L. A. Turski, and A. Zippelius, Phys. Rev. Lett. 58, 1656 (1987); J. P. Sethna, Europhys. Lett. 6, 529 (1988); J. P. Sthena, J. D. Shore, and M. Huang, Phys. Rev. B 44, 4943 (1991); X. Y. Xia and P. G. Wolynes, J. Phys. Chem. B 105, 6570 (2001).

${ }^{4}$ P. G. Debenenedetti, Metastable Liquids: Concepts and Principles (Princeton University Press, Princeton, NJ, 1996), Chap. 4.4, and references therein.

${ }^{5}$ W. Kauzmann, Chem. Rev. (Washington, D.C.) 43, 219 (1948).

${ }^{6}$ G. Adam and J. H. Gibbs, J. Chem. Phys. 43, 139 (1965).

${ }^{7}$ J. H. Gibbs and E. A. DiMarzio, J. Chem. Phys. 28, 373 (1958)

${ }^{8}$ M. Goldstein, J. Chem. Phys. 51, 3728 (1969).

${ }^{9}$ F. H. Stillinger and J. Hodgdon, Phys. Rev. E 50, 2064 (1994); 53, 2995 (1996); F. H. Stillinger, J. Chem. Phys. 89, 6461 (1988), and references therein; P. G. Debenedeti and F. H. Stillinger, Nature (London) 410, 256 (2001).

${ }^{10}$ S. Sastry, Nature (London) 409, 164 (2001); R. J. Speedy, J. Phys. Chem. 103, 4060 (1999); C. T. Moynihan and C. A. Angell, J. Non-Cryst. Solids 274, 131 (2000); S. Sastry, J. Phys.: Condens. Matter 12, 6515 (2000); T. F. Middleton and D. J. Wales, Phys. Rev. B 64, 024205 (2001); R. A. Denny, D. R. Reichman, and J. P. Bouchaud, Phys. Rev. Lett. 90, 025503 (2003).

${ }^{11}$ B. Doliwa and A. Heuer, Phys. Rev. Lett. 80, 4915 (1998).

${ }^{12}$ R. Yamamoto and A. Onuki, Phys. Rev. Lett. 81, 4915 (1998); A. Onuki and Y. Yamamoto, J. Non-Cryst. Solids 235-237, 34 (1998); A. Onuki and Y. Yamamoto, Int. J. Mod. Phys. C 10, 1553 (1999).

${ }^{13}$ D. Perera and P. Harrowell, J. Non-Cryst. Solids 235-237, 314 (1998); D. Perera and P. Harrowell, Phys. Rev. E 59, 5721 (1999); 54, 1652 (1996); H. Fynewever and P. Harrowell, J. Phys.: Condens. Matter 12, 6305 (2000).

${ }^{14}$ T. Muranaka and Y. Hiwatari, Phys. Rev. E 51, R2735 (1995); Y. Hiwatari and T. Muranaka, J. Non-Cryst. Solids 235-237, 19 (1998).

${ }^{15}$ A. I. Mel'cuk, R. A. Ramos, H. Gould, W. Klein, and R. D. Mountain, Phys. Rev. Lett. 75, 2522 (1995); G. Johnson, A. I. Mel'cuk, H. Gould, W. Klein, and R. D. Mountain, Phys. Rev. E 57, 5707 (1998).

${ }^{16}$ M. Dzugutov, S. I. Simdyankin, and F. H. M. Zetterling, Phys. Rev. Lett. 89, 195701 (2002).

${ }^{17}$ W. Kob, C. Donati, P. H. Poole, S. J. Plimpton, and S. C. Glotzer, Phys. Rev. Lett. 79, 2827 (1997).

${ }^{18}$ F. Sciortino, W. Kob, and P. Tartaglia, Phys. Rev. Lett. 83, 3214 (1999).

${ }^{19}$ B. Coluzzi, Ph.D. thesis, University of Roma La Sapienza, 1999; B. Coluzzi, M. Mezard, P. Verrocchio, and G. Parisi, J. Chem. Phys. 111, 9039 (1999).

${ }^{20}$ A. Scala, F. W. Starr, E. La Nave, F. Sciortino, and H. E. Stanley, Nature (London) 406, 166 (2000).

${ }^{21}$ K. Schmidt-Rohr and H. W. Spiess, Phys. Rev. Lett. 66, 3020 (1991).

${ }^{22}$ I. Chang and H. Sillescu, J. Phys. Chem. B 101, 8794 (1997).

${ }^{23}$ R. Böhmer et al., J. Non-Cryst. Solids 235-237, 1 (1998).

${ }^{24}$ M. T. Cicerone, F. R. Blackburn, and M. D. Ediger, Macromolecules 28, 8224 (1995); M. T. Cicerone and M. D. Ediger, J. Chem. Phys. 104, 7210
(1996); F. R. Blackburn et al., J. Non-Cryst. Solids 172-174, 256 (1994); S. F. Swallen, P. A. Bonvallet, R. J. McMahon, and M. D. Ediger, Phys. Rev. Lett. 90, 015901 (2003).

${ }^{25}$ D. B. Hall, A. Dhinojwala, and J. M. Torkelson, Phys. Rev. Lett. 79, 103 (1997).

${ }^{26}$ A. Heuer et al., Phys. Rev. Lett. 95, 2851 (1995).

${ }^{27}$ L. Andreozzi, A. Di Schino, M. Giordano, and D. Leporini, Europhys. Lett. 38, 669 (1997)

${ }^{28}$ R. Richert, Chem. Phys. Lett. 199, 355 (1992); R. Richert, F. Stickel, R. S. Fee, and M. Maroncelli, ibid. 229, 302 (1994); H. Wendt and R. Richert, Phys. Rev. E 61, 1722 (2000); M. Yang and R. Richert, J. Chem. Phys. 115, 2676 (2001); R. Richert, J. Non-Cryst. Solids 172-174, 209 (1994).

${ }^{29}$ E. V. Russell and N. E. Israeloff, Nature (London) 408, 695 (2000).

${ }^{30}$ B. Schiener, R. Böhmer, A. Loidl, and R. V. Chamberlin, Science 274, 752 (1996); B. Schiener, R. V. Chamberlin, G. Diezemann, and R. Böhmer, J. Chem. Phys. 107, 7746 (1997).

${ }^{31}$ M. Russina, F. Mezei, R. Lechner, S. Longeville, and B. Urban, Phys. Rev. Lett. 84, 3630 (2000).

${ }^{32}$ E. Weeks, J. C. Crocker, A. C. Levitt, A. Schofield, and D. A. Weitz, Science 287, 627 (2000); E. R. Weeks and D. A. Weitz, Phys. Rev. Lett. 89, 095704 (2002).

${ }^{33}$ W. K. Kegel and A. van Blaaderen, Science 287, 290 (2000).

${ }^{34}$ A. van Blaaderen and P. Wiltzius, Science 270, 1177 (1995).

${ }^{35}$ L. A. Deschenes and D. A. Vanden Bout, Science 292, 255 (2001).

${ }^{36}$ A. H. Marcus, J. Schofield, and S. A. Rice, Phys. Rev. E 60, 5725 (1999); 61, 7260 (2000).

${ }^{37}$ H. Sillescu, J. Non-Cryst. Solids 243, 81 (1999), and references therein.

${ }^{38}$ M. D. Ediger, Annu. Rev. Phys. Chem. 51, 99 (2000).

${ }^{39}$ R. Richert, J. Phys.: Condens. Matter 14, R703 (2002)

${ }^{40}$ X. Qiu and M. D. Ediger, J. Phys. Chem. B 107, 459 (2003); S. A. Reinsberg, X. H. Qiu, M. Wilhelm et al., J. Chem. Phys. 114, 7299 (2001)

${ }^{41}$ E. Leutheusser, Phys. Rev. A 29, 2765 (1984); U. Bengtzelius, W. Götze, and A. Sjölander, J. Phys. C 17, 5915 (1984); W. Götze and L. Sjögren, Rep. Prog. Phys. 55, 241 (1992); W. Götze and L. Sjögren, Chem. Phys. 212, 47 (1996); W. Götze and L. Sjögren, Transp. Theory Stat. Phys. 24, 801 (1995).

${ }^{42}$ R. L. Leheny, N. Menon, S. R. Nagel, D. L. Price, K. Suzuya, and P. Thiyagarajena, J. Chem. Phys. 105, 7783 (1996).

${ }^{43}$ C. Bennemann, C. Donati, J. Baschnagel, and S. C. Glotzer, Nature (London) 399, 246 (1999)

${ }^{44}$ C. Donati, S. C. Glotzer, P. H. Poole, W. Kob, and S. J. Plimpton, Phys. Rev. E 60, 3107 (1999).

${ }^{45}$ C. Donati, P. H. Poole, and S. C. Glotzer, Phys. Rev. Lett. 82, 6064 (1999).

${ }^{46}$ C. Donati, J. F. Douglas, W. Kob, P. H. Poole, S. J. Plimpton, and S. C. Glotzer, Phys. Rev. Lett. 80, 2338 (1998).

${ }^{47}$ S. C. Glotzer, J. Non-Cryst. Solids 274, 342 (2000).

${ }^{48}$ A. Onuki and Y. Yamamoto, Phys. Rev. E 58, 3515 (1998).

${ }^{49}$ M. Hurley and P. Harrowell, Phys. Rev. E 52, 1694 (1995).

${ }^{50}$ B. Doliwa and A. Heuer, Phys. Rev. E 61, 6898 (2000).

${ }^{51}$ R. D. Mountain, Supercooled Liquids American Chemical Society Symposium 676, 122 (1997); J. Chem. Phys. 102, 5408 (1995).

${ }^{52}$ P. Scheindler, W. Kob, K. Binder, and G. Parisi, Philos. Mag. B 82, 283 (2002); P. Scheindler, W. Kob, and K. Binder, Europhys. Lett. 59, 701 (2002).

${ }^{53}$ G. Parisi, J. Phys. Chem. B 103, 4128 (1999).

${ }^{54}$ J. P. Garrahan and D. Chandler, Phys. Rev. Lett. 89, 035704 (2002); J. P. Garrahan and D. Chandler, cond-mat/0301287.

${ }^{55}$ see, e.g., G. H. Fredrickson and H. C. Andersen, Phys. Rev. Lett. 53, 1244 (1984); J. Jäckle and S. Eisinger, Z. Phys. B: Condens. Matter 84, 115 (1991); F. Ritort and P. Sollich, cond-mat/0210382.

${ }^{56}$ S. C. Glotzer and C. Donati, J. Phys.: Condens. Matter 11, A285 (1999).

${ }^{57}$ S. C. Glotzer, Phys. World 13, 22 (2000).

${ }^{58}$ C. Dasgupta, A. V. Indrani, S. Ramaswamy, and M. K. Phani, Europhys. Lett. 15, 307 (1991) [Addendum: Europhys. Lett. 15, 467 (1991)].

${ }^{59}$ S. Franz, C. Donati, G. Parisi, and S. C. Glotzer, Philos. Mag. B 79, 1827 (1999); C. Donati, S. Franz, S. C. Glotzer, and G. Parisi, J. Non-Cryst. Solids 307, 215 (2002).

${ }^{60}$ S. C. Glotzer, V. N. Novikov, and T. B. Schrøder, J. Chem. Phys. 112, 509 (2000).

${ }^{61}$ N. Lačević, F. W. Starr, T. B. Schrøder, V. N. Novikov, and S. C. Glotzer, Phys. Rev. E 66, 030101 (2002).

${ }^{62}$ G. Wahnström, Phys. Rev. A 44, 3752 (1991). 
${ }^{63}$ T. B. Schrøder, "Hopping in disordered media: A model glassformer and a hopping model," cond-mat/0005127.

${ }^{64}$ Steve Plimpton, Sandia National Labs, www.cs.sandia.gov/ sjplimp

${ }^{65}$ T. B. Schrøder, S. Sastry, J. C. Dyre, and S. C. Glotzer, J. Chem. Phys. 112, 9834 (2000).

${ }^{66}$ T. B. Schrøder and J. C. Dyre, J. Non-Cryst. Solids 235-237, 331 (1998).

${ }^{67}$ J. P. Hansen and I. R. McDonald, Theory of Simple Liquid, (Academic, New York, 1986).

${ }^{68}$ We calculated the average total energy and the temperature as a function of block size and compared with the total energy and temperature for the entire run. We choose the length of the block whose average total energy and temperature are the same as for the entire run and calculate standard deviation and uncertainty according to the book by Andrew R. Leach, Molecular Modeling, Principles and Applications (Longman, Edinburgh Gate, 1996), p. 305.

${ }^{69}$ P. Allegrini, J. F. Douglas, and S. C. Glotzer, Phys. Rev. E 60, 5714 (1999).

${ }^{70}$ S. Franz and G. Parisi, cond-mat/9804084.

${ }^{71}$ S. Franz and G. Parisi, J. Phys.: Condens. Matter 12, 6335 (2000).

${ }^{72}$ T. R. Kirkpatrick and P. G. Wolynes, Phys. Rev. A 35, 3072 (1987); 36, 852 (1987); T. R. Kirkpatrick and D. Thirumalai, Phys. Rev. B 36, 5388 (1987); Transp. Theory Stat. Phys. 24, 927 (1995).

${ }^{73}$ A. Crisanti, H. Horner, and H. J. Sommers, Z. Phys. B: Condens. Matter 92, 257 (1993).

${ }^{74} \mathrm{~A}$ review of the $p$-spin model can be found in A. Barrat, cond-mat/9701031 (unpublished).

${ }^{75}$ Moreover, caging is still somewhat of a quantitatively ill-defined concept, and can be defined both as a particle whose neighbors prevent its motion on the scale of an interparticle spacing, and a particle whose local environment remains static. A particle that is uncaged is one whose environment has significantly changed, and who has moved a significant distance.

${ }^{76}$ S. C. Glotzer, C. Donati, and P. H. Poole, Computer Simulation Studies in Condensed-Matter Physics XI, Springer Proceedings in Physics, edited by D. P. Landau and H.-B. Schüttler (Springer-Verlag, Berlin Heidelberg, 1999), Vol. 84, p. 212.
${ }^{77}$ Strictly speaking, a simulation in the grand canonical ensemble would instead yield a small but nonzero $\chi_{4}(t)$ value at early times.

${ }^{78}$ In Ref. 61 we investigated the function $g_{4}^{*}(r, t)$ defined by Eq. (17). We note that $g_{4}(r, t)$ and $g_{4}^{*}(r, t)$ correspond to $\chi_{4}(t)$ and $\chi_{4}^{*}(t)$, respectively, where $\chi_{4}^{*}(t)$ is obtained from the following simplification of Eq. (16) by means of Eq. (17): $\chi_{4}(t)=\beta\langle Q(t) / N\rangle^{2} \int d^{3} r g_{4}^{*}(r, t) \equiv\langle Q(t) / N\rangle^{2} \chi_{4}^{*}(t)$. We compare the time dependence of both $\chi_{4}(t)$ and $\chi_{4}^{*}(t)$, and find that the peak time of $\chi_{4}^{*}(t)$ is shifted to later times compared to the peak time of $\chi_{4}(t)$ for all temperatures. As a consequence, the peak time in the correlation length calculated from $g_{4}^{*}(r, t)$ is shifted to later times than $t_{4}^{\max }$ for all temperatures. Both $g_{4}(r, t)$ and $g_{4}^{*}(r, t)$ are correlation functions of overlapping particles and their correlation lengths are the same if the "envelope" method is used to estimate those lengths. In the envelope method we fit peaks of $g_{4}^{*}(r, t)$ over a certain range to the exponential function $y(r)=a \times \exp \left(-r / \xi_{4}^{\text {env }}(t)\right)$ (see Ref. 61). The common term in $g_{4}(r, t)$ and $g_{4}^{*}(r, t)$ is $g_{4}^{o l}(r, t)$, and it is the correlation length of this term that we now investigate.

${ }^{79}$ H. E. Stanley, Introduction to Phase Transitions and Critical Phenomena (Oxford University Press, New York, 1971).

${ }^{80}$ T. F. Coleman and Y. Li, Math. Program. 67, 189 (1994).

${ }^{81}$ W. Kob and H. C. Andersen, Phys. Rev. E 52, 4134 (1995); 51, 4626 (1995); F. Sciortino and W. Kob, Phys. Rev. Lett. 86, 648 (2001).

${ }^{82}$ S. J. Pitts, T. Young, and H. C. Andersen, J. Chem. Phys. 113, 8671 (2000).

${ }^{83}$ M. Foley and P. Harrowell, J. Chem. Phys. 98, 5069 (1993).

${ }^{84}$ S. C. Glotzer, N. Jan, T. Lookman, A. B. MacIsaac, and P. H. Poole, Phys. Rev. E 57, 7350 (1998).

${ }^{85}$ Y. Gebremichael, T. B. Schrøder, F. W. Starr, and S. C. Glotzer, Phys. Rev. E 64, 051504 (2001).

${ }^{86}$ R. van Zon and J. Schofield, Phys. Rev. E 65, 011106 (2002).

${ }^{87}$ R. van Zon and J. Schofield, Phys. Rev. E 65, 011107 (2002). 